\title{
Ecological and social pressures interfere with homeostatic sleep regulation in the wild
}

Loftus JC ${ }^{\mathrm{abcd}}$, Harel R ${ }^{\mathrm{b}}$, Nuñez $\mathrm{CL}^{\mathrm{bd}} \&$ Crofoot $\mathrm{MC}^{\mathrm{abcd}}$

a Department of Anthropology, University of California at Davis, Davis, CA 95616

b Department for the Ecology of Animal Societies, Max Planck Institute of Animal Behavior, Konstanz, Germany 78467

c Department of Biology, University of Konstanz, Konstanz, Germany 78464

d Centre for the Advanced Study of Collective Behaviour, University of Konstanz, Konstanz, Germany 78464

Corresponding author:

Carter Loftus

1 Shields Avenue

Davis, CA 95616

$+49.159 .068 .45928$

jcloftus@ucdavis.edu

Keywords: sleep, homeostasis, olive baboon, predation risk, social behavior, biotelemetry 


\section{Abstract}

Sleep is fundamental to the health and fitness of all animals. The physiological importance of sleep is underscored by the central role of homeostasis in determining sleep investment - following periods of sleep deprivation, individuals experience longer and more intense sleep bouts. Yet, most studies of sleep have been conducted in highly controlled settings, disconnected from the ecological and social context that may exert pressures on sleep patterns in conflict with homeostatic regulation. Using tri-axial accelerometry and GPS to track the sleep patterns of a group of wild baboons (Papio anubis) at multiple temporal and spatial scales, we found that ecological and social pressures indeed interfere with homeostatic sleep regulation. Baboons sacrificed time spent sleeping when in less familiar locations and when sleeping in proximity to more group-mates, regardless of how much they had slept the prior night or how much they had physically exerted themselves the preceding day. Moreover, we found that the collective dynamics characteristic of social animal groups persist into the sleep period, as baboons exhibited synchronized patterns of waking throughout the night, particularly with nearby group-mates. Thus, for animals whose fitness depends critically on avoiding predation and developing social relationships, maintaining sleep homeostasis may be only secondary to remaining vigilant when sleeping in risky habitats and interacting with group-mates during the night. Our results highlight the importance of studying sleep in ecologically relevant contexts, where the adaptive function of sleep patterns directly reflect the complex trade-offs that have guided its evolution.

\section{Introduction}

Sleep is an important and understudied facet of animal lives, with every species, from honey bees to humans, allocating a portion of every day to this period of rest (Cirelli \& Tononi, 2008). The universality of sleep reflects its central role in important physiological processes, including memory consolidation, support of the central nervous system, energy conservation and physical restoration (Chowdhury \& Shafer, 2020; Gangwisch, 2014; Stickgold, 2005; Vyazovskiy, 2015). Accordingly, failure to meet daily sleep demand has health consequences (Basner et al., 2013), with potentially fatal repercussions of extreme sleep deprivation (Rechtschaffen \& Bergmann, 2002). The physiological need for sleep is emphasized by its homeostatic control - after periods of insufficient sleep or extreme physical exertion, individuals experience particularly long and intense bouts of sleep (Kitamura et al., 2016). Decades of sleep research have consistently implicated homeostasis as a primary determinant of sleep patterns, such that homeostatic regulation has become an important criterion in the very definition of sleep (Siegel, 2008).

A strong focus on studying sleep in the laboratory or at the bedside, although revealing much about the physiology of sleep, has inherently overlooked the ecological pressures that drive the regulation and evolution of sleep (Rattenborg et al., 2017; Reinhardt, 2020). In the natural world, the significance of sleep extends beyond its direct physiological impacts. Sleeping animals typically cannot engage in other behaviors that are important to their survival (but see Rattenborg et al., 2016), 
and investing in sleep when environmental forces render vigilance and activity particularly important may impose substantial costs to wild animals. In addition to preventing animals from foraging, searching for mating opportunities, defending territories, and caring for young, sleep leaves animals in a state of extreme inattention, and thus highly vulnerable to their predators (Lima et al., 2005). The evolution of sleep and its manifestation in the wild may therefore be driven by a complex balance between the physiological need for sleep and ecological costs imposed on sleeping animals.

For gregarious animals, the balance between the costs and benefits of sleep may be further modulated by the social environment. However, even the most basic aspects of sleeping with conspecifics, such as whether the social context facilitates or constrains sleep, remain unknown (Karamihalev et al., 2019). Sleeping in a social context could alter the costs of sleep - the sentinel hypothesis suggests that staggering the timing of sleep bouts in a group can collectively maintain both high quality sleep and high levels of anti-predator vigilance (Samson et al., 2017; Snyder, 1966). Sleeping in a group may therefore facilitate an individual's ability to fulfill its physiological requirements for sleep by reducing the risk of doing so. Alternatively, social dynamics may actually inhibit investment in sleep. Sleep may present social opportunity costs, causing individuals to sacrifice sleep in order to spend more time actively engaging with group-mates. Additionally, proximity to group-mates may cause cascading disruptions initiated by short periods of wakeful activity of neighboring individuals. Thus, sleeping in close proximity to conspecifics may potentially be accompanied by both costs and benefits for an individual's ability to obtain sufficient sleep, and discovering how these potential costs and benefits are actually realized will shed light on the forces that have guided sleep adaptations in social animals.

To understand how group-living animals navigate tradeoffs between their physiological need for sleep and the ecological and social pressures that shape the costs and benefits associated with this biological imperative, we investigated the factors shaping sleep patterns of wild olive baboons (Papio anubis). Baboons live in stable multi-male, multi-female groups of up to 100 individuals (Cheney \& Seyfarth, 2008), and during the night, they seek safety in trees and rock outcroppings (Altmann \& Altmann, 1970; Busse, 1980). Despite seeking refuge in these sleep sites, baboons remain particularly vulnerable to nighttime predation from leopards, which represents the single largest source of mortality for adult baboons (Cheney et al., 2004; Cowlishaw, 1994; Isbell et al., 2018). Baboons must therefore navigate the tradeoff between investing in sleep and maintaining anti-predator vigilance. As a highly gregarious animal whose fitness depends heavily on their social relationships (Silk et al., 2009), baboons must also balance their time spent sleeping with their investment in social interactions, as time constraints during the day limit their ability to build and maintain their relationships (Dunbar, 1992).

We simultaneously tracked the activity of 26 wild olive baboons from the same group using collars fitted with GPS sensors and tri-axial accelerometers to understand how baboons manage their competing nighttime priorities. Accelerometer-based sleep classification has shown an impressive 
ability to detect and monitor sleep behavior across taxa (Ancoli-Israel et al., 2003; de Souza et al., 2003; Hoffmann et al., 2012; Ladha \& Hoffman, 2018; Malungo et al., 2021; Qin et al., 2020), and is now commonly used to assess sleep in both humans (e.g. Jones et al., 2019; Patel et al., 2017) and non-human animals (e.g. Gravett et al., 2017; Reinhardt, 2020; Samson et al., 2018). Validation studies comparing performance of this non-invasive method to polysomnogaphy - the gold standard in sleep research — generally show high accuracy (78-90\%; Ancoli-Israel et al., 2003; Kanady et al., 2011; Malungo et al., 2021; Shambroom et al., 2012), although concerns remain about the ability of movement-based methods to distinguish sleep from resting wakefulness (Ancoli-Israel et al., 2003; de Souza et al., 2003), and results must be evaluated with these caveats in mind. For this study, we adapted a well-validated sleep classification algorithm used in human research (van Hees et al., 2015; van Hees et al., 2018) and validated its ability to detect sleep in wild baboons. We then used this algorithm to describe the sleep patterns of members of our study group over a period of a month (Table S1). We used these data to assess the influence of homeostatic regulation on the pattern of sleep and wake bouts within nights, as well as the duration of sleep across nights. We compared this influence to that of the location in which individuals slept (both within the sleep site as well as between distinct sleep sites) and their local social environment, both of which may exert pressures on sleep behavior in the wild that conflict with the maintenance of homeostasis.

\section{Results}

The diel pattern of activity in wild baboons, as reflected by accelerometry data, reveals a clear monophasic sleep pattern, with individuals active during the day and inactive at night (Fig. 1B). To derive metrics of sleep (sleep onset time, awakening time, total sleep time, sleep period duration, sleep efficiency), we calculated the log of the vectorial dynamic body acceleration (VeDBA), a widely-used measure of overall movement activity (Qasem et al., 2012), from 36 calendar days or 354 baboon-nights. Sleep onset occurred $53.0 \pm 1.7$ (mean $\pm \mathrm{SE}$ ) minutes prior to the end of evening astronomical twilight, and baboons awoke $35.9 \pm 1.7$ minutes after the beginning of morning astronomical twilight (Fig. 1A,C). The duration of the sleep period - the period between sleep onset and awakening - was $11.0 \pm 0.04$ hours on average. Within the sleep period, baboons slept for an average of $9.2 \pm 0.04$ hours (total sleep time), displaying an average sleep efficiency of $85.0 \% \pm$ $0.2 \%$.

\section{[Figure 1]}

Due to high correlation of total sleep time with onset time, awakening time, sleep period duration, and sleep efficiency (Table S2), we focused the majority of our analyses on total sleep time. Individuals differed in their total sleep time, and much of this variation reflected differences between the sexes and variation across age categories. Males slept an average of 20 minutes longer than females (Linear Mixed Model (LMM): standardized estimate [95\% credible interval lower bound, 95\% credible interval upper bound]: 0.44 [-0.04, 0.92]), while juveniles and subadults slept, on 
average, 15 minutes less than adults (LMM: juveniles: -0.32 [-1.12, 0.50]; subadults: -0.32 [-0.80, $0.14])$.

The maintenance of homeostasis was not a strong driver of sleep patterns (Fig. 2). After sleeping poorly (low total sleep time), baboons did not 'catch up' by napping more on the following day (Table S6; LMM: 0.05 [-0.08, 0.18]) or sleeping longer the following night (Fig. 2A; LMM: 0.00 $[-0.14,0.13])$. However, baboons did sleep less following days on which they spent more time napping (Fig. 2B; LMM: -0.18 [-0.36, -0.01]). For every minute spent napping, baboons spent 0.6 fewer minutes sleeping the following night. Baboons' sleep duration was uninfluenced by their physical exertion during the day, as measured by either the distance they traveled (Fig. 2C; LMM: 0.02 [-0.18, 0.15],) or their cumulative daytime VeDBA (Table S5; LMM: -0.12 [-0.33, 0.12]). In humans, homeostatic regulation of sleep manifests within, as well as between nights: sleep wanes and wakeful activity increases over the course of the sleep period as individuals gradually fulfill their sleep requirements (Winnebeck et al, 2018). Baboons, in contrast, did not experience this increase in wakefulness as the night progressed, despite exhibiting cyclic sleep patterns that are otherwise similar to patterns of human sleep (Fig. 2D; GAMM: $\mathrm{r}^{2}{ }_{\text {adj }}=0.008, \mathrm{~F}_{(8.741)}=89.16, \mathrm{p}<1 \times 10^{-15}$ ).

[Figure 2]

The location where baboons slept had a strong influence on sleep duration, with individuals sleeping less when spending the night in less familiar locations. For the first 21 nights of the study, group members slept at the same site, distributed across ten adjacent yellow fever (Acacia xanthophloea) trees (Fig. 3A). Individuals showed high fidelity to particular sleep trees (Fig. S3; onetailed two-sample Kolmogorov-Smirnov test: $\left.\mathrm{p}<1.0 \times 10^{-9}\right)$, returning each night to one or a small set of the available trees populated by the group. Not only did the choice of tree itself influence sleep duration (Table S3 - S4, Fig. S4), but the individual's familiarity with their selected tree impacted how much they slept. Baboons slept longer in trees to which they showed higher fidelity (Fig. 3C; LMM: $0.20[0.05,0.36])$, with individuals sleeping up to 33.3 minutes longer in the tree to which they showed highest fidelity than in the tree to which they showed lowest fidelity. Following a failed leopard attack on the $21^{\text {st }}$ night of the study, the group moved to a less commonly used sleep site 1.5 km away (Fig. 3B). They remained at this sleep site for three nights before returning to sleep at their main sleep site. While the baboons showed no substantial decrease in their sleep duration on the night of the leopard attack (Fig. 3D, Fig. S7; LMM: -0.25 [-0.87, 0.39]), they slept 72 minutes less, on average, upon moving to the less familiar sleep site (Fig. 3D, Fig. S7; LMM: -1.55 [-2.18, -0.91]). This decrease in total sleep time following the change in sites was limited to the first night in the new sleep site, after which sleep durations returned to normal (Fig. 3D; Fig. S7).

[Figure 3]

Sleeping in a social context also impacted sleep duration, as group-mates disrupted each other's rest during the night. Contrary to predictions of the sentinel hypothesis, the proportion of the night in which at least one individual was awake was significantly less than expected by chance (Fig. 
4A; Fisher's exact test: $\mathrm{p}<0.0001)$, suggesting that, rather than staggering periods of nocturnal wakefulness, group-mates were actually synchronized in their sleep-wake patterns throughout the night. Confirming this synchronization, we found that a significantly greater proportion of the group exhibited the same simultaneous behavior, either being asleep or awake, than expected (Fig. 4B; Fisher's exact test: $\mathrm{p}<0.0001)$. Group members showed a unique pattern of synchronized sleep and wake bouts each night, and thus, synchronization was not a spurious result of a stereotyped schedule of activity that happened to be consistent across baboons and across nights (Fig. S10; Fisher's exact test: $\mathrm{p}<0.0001)$. Moreover, pairs of baboons showed more synchronization when sleeping in the same tree than when sleeping in different trees (Fig. 4C; LMM: 0.56 [0.47-0.64]), which suggested that sleeping individuals may awaken in response to the activity of group-mates in their local environment, or that external disruptions in the local environment may simultaneously waken all group members in the vicinity. To distinguish between these potential explanations, we tested the influence of the number of group-mates in an individual's local environment on their total sleep time, and found that individuals slept less when sharing their sleeping tree with more group-mates (Fig. 4D; LMM: $-0.51[-0.85,-0.16])$. Each additional tracked group-mate in a tree resulted in a 4.0-minute decrease in total sleep time. Taken together, these results indicate that synchronization resulted from social disruptions of sleep.

\section{[Figure 4]}

We found no influence of moon phase or the minimum ambient temperature during the night on baboon sleep duration (LMM: Moon phase: 0.05 [-0.12, 0.22]; Temperature: -0.03 [-0.21, 0.13]).

\section{Discussion}

In this study, we demonstrate that the ecological and social demands that animals experience in the natural world disrupt the widely-reported homeostatic regulation of sleep. We show that while baboons sleep less in unfamiliar environments and their sleep is disrupted by the activity of groupmates, their recent history of sleep and physical exertion has only a limited role in influencing sleep behavior. Because baboons are highly vulnerable to nocturnal predation (Busse, 1980; Cheney et al., 2004; Isbell et al., 2018) and because they experience fitness benefits from maintaining strong social bonds (Silk et al., 2009), sacrificing sleep to maintain alertness in novel environments and to remain close to group-mates may represent critical adaptations. Our results highlight the tradeoffs that groupliving animals navigate when investing in sleep in the wild, and in doing so, challenge the centrality of the role that homeostasis has played in shaping sleep patterns in the environment in which sleep evolved. Decades of research in the laboratory and at the bedside have implicated homeostatic regulation as a key driver of sleep patterns, with the sleep rebound that follows periods of deficit facilitating the maintenance of a physiologically required amount of sleep (Amlaner et al., 2009). However, sleep studies have traditionally investigated sleep in highly controlled environments, where the costs of investing in sleep are largely absent. Our findings suggest that, in the natural world, 
"sleep need" may be a relatively flexible concept, with variation in sleep investment driven as much by the opportunity costs of sleep as by its physiological benefits.

There are substantial opportunity costs of devoting a significant portion of every day to sleeping. Sleeping animals are highly vulnerable to predation (Lima et al., 2005), and our results suggest that individuals sleep less when the risk of predation is particularly high. Baboon group members showed high fidelity to particular locations within their main sleep site, and individuals sacrificed sleep both when sleeping in trees to which they did not show high fidelity as well as upon moving to a new, less familiar sleep site following a leopard attack. Given that predation risk tends to be greater in unfamiliar locations (Forrester et al., 2015; Gehr et al., 2020; Yoder et al., 2004), baboons appear to trade sleep for vigilance according to the current risk of predation. Notably, however, we did not find that baboons decreased their investment in sleep on the night of leopard attack. This surprising result may reflect leopards' disinclination, as stealth hunters, to launch repeated attacks (Hayward et al., 2006; Lin et al., 2020), or indicate that baboons perceive uncertainty in the level of risk as potentially more dangerous than a confirmed threat.

Engaging in sleep precludes investment in a variety of behaviors, in addition to anti-predator vigilance, that are important to fitness (Aulsebrook et al., 2016; Lesku et al., 2012; Lima et al., 2005; Roth et al., 2010). Consistent with our results, recent studies in ecologically-relevant contexts have revealed that animals forego sleep when ecological demands increase the associated opportunity costs. For example, while engaging in long, non-stop flights, great frigatebirds reduced the amount they slept by 92.7\%, without apparent physiological consequences (Rattenborg et al., 2016). Similarly, northern fur seals (Callorhinus ursinus) experience extended suppression of rapid eye movement (REM) sleep - also shown in the laboratory to be homeostatically regulated (Dement, 1960; Shiromani et al., 2000) - when they migrate in the open ocean for several months (Lyamin et al., 2018). Moreover, the seals do not experience a rebound in their REM sleep following this period of deficit (Lyamin et al., 2018). Male pectoral sandpipers greatly reduce their time spent sleeping during their short and intense mating season, and males that slept less actually experienced higher reproductive success (Lesku et al., 2012). Thus, across contexts and taxa, ecological pressures appear to supersede the maintenance of sleep homeostasis in the wild.

Recent technological advances allowing for the use of polysomnography - the gold standard for recording sleep - have played an important role in revealing the ecological tradeoffs that wild animals face when navigating decisions about when, where, and how to sleep (Davimes et al., 2018; Lesku et al., 2011, 2012; Rattenborg et al., 2008, 2016; Scriba et al., 2013; Voirin et al., 2014). Although these advances hold great promise for wider application in the future, the invasive nature of polysomnography unfortunately limits its current use to taxa whose daily activities do not interfere with electrodes implanted either subdermally or inter-cranially. Because baboons are highly dexterous and engage in frequent allogrooming, we were unable to apply this gold standard, and instead, resorted to an alternate method in order to ask and answer important questions about the ecology of 
sleep in a wild social primate. Accelerometer-based sleep classification - a tool already used to investigate sleep across terrestrial (human: Jones et al., 2019; Patel et al., 2017; non-human: Bäckman et al., 2017; Davimes et al., 2018; Gravett et al., 2017; Lesku et al., 2011; Malungo et al., 2021; Qin et al., 2020; Reinhardt et al., 2019; Reyes et al., 2021; Samson et al., 2018; Sellers \& Crompton, 2004; Sri Kantha \& Suzuki, 2006; Suzuki et al., 2018) and marine taxa (Miller et al., 2008; Mitani et al., 2010; Wright et al., 2017) - offered a valid alternative to polysomnography. We note that the use of accelerometry can introduce biases in sleep monitoring, typically by overestimating total sleep time as a result of an inability to distinguish resting wakefulness from sleep (Ancoli-Israel et al., 2003; de Souza et al., 2003). However, if these biases are considered during the interpretation of results, the use of accelerometry to investigate sleep provides an immediate opportunity to shed light on how diverse species balance their physiological sleep requirements with ecological opportunity costs that vary according to natural history, trophic level, community composition, climate, and local environment. Further, the relative ease of accelerometer deployment, and its prevalence in ecological research, changes the scale at which sleep behavior can be studied, enabling the simultaneous and long-term monitoring of sleep at the population level. This rescaling of sleep research creates many new opportunities, one of which is the ability to record sleep in the majority of social group members and thus explore an exciting new scientific frontier: the collective dynamics of sleep.

Using accelerometry to track the sleep patterns of nearly an entire group of wild baboons, we demonstrated the importance of the social environment in shaping the sleep patterns of group-living animals. Contrary to the predictions of the sentinel hypothesis (Samson et al., 2017; Snyder, 1966), periods of nocturnal wakefulness of group members were not staggered, but rather synchronized, particularly with nearby group-mates. Baboons also slept less when in close proximity to a greater number of group-mates. Taken together, these results suggest that group-mates disrupt each other's sleep. Social disruptions may result from group-mates actively interacting with each other during the night. Gregarious animals often invest substantially in building and maintaining social relationships with their group-mates (Ward \& Webster, 2016), and these bonds can prove essential to their fitness (Cameron et al., 2009; Campos et al., 2020; Frère et al., 2010; Riehl \& Strong, 2018; Silk et al., 2009). Because animals have limited time to devote to maintaining their social bonds during the day (Dunbar, 1992), they may actively sacrifice sleep in order to invest in these relationships at night. Alternatively, social animals may wake in response to the periodic waking and repositioning of their group-mates during the night, and thus, socially-disrupted sleep may be an inherent by-product of sleeping in a group. Simply remaining in a cohesive group may therefore present a challenge to obtaining sufficient sleep.

Social animals may jeopardize sleep homeostasis to maintain cohesion with their conspecifics because remaining in close proximity to their group-mates during the sleep period could prove essential to their fitness. Individuals likely benefit from the dilution of predation risk that is achieved through group cohesion, particularly when they are sleeping and thus highly vulnerable to predators 
313 (Lehtonen \& Jaatinen, 2016). Collective vigilance may also reduce the risk of predation for group

314 members. Even in the absence of collective vigilance optimization via non-randomly staggered

315 wakefulness, the proportion of the night with at least one group member awake is still likely to be

316 substantially greater than any particular individual's investment in vigilance. In our study, at least one

317 individual in the group was awake for $394 \pm 11$ minutes $(82 \% \pm 2 \%)$ from 21:00 to 05:00, although

318 each individual was only awake for $79 \pm 1$ minutes $(16 \% \pm 0.2 \%)$ of the same period. Samson and

319 colleagues (2017) found high levels of collective vigilance during the night in a group of Hadza

320 hunter-gatherers, and they suggest that this collective vigilance may facilitate higher intensity sleep

321 (Samson \& Nunn, 2015). Because accelerometry cannot measure sleep intensity, we were unable to

322 test whether collective vigilance allowed individuals sleeping close to group-mates to experience

323 more intense, albeit shorter, sleep. Future studies leveraging advances in polysomnography (i.e. EEG)

324 that may eventually allow its application in wild social animals could enable a test of this possibility.

325 Unexpectedly, we found that adult baboons slept longer than subadults and juveniles, and

326 males slept longer than females. This contrasts with previous research that found age differences in

327 sleep patterns linked to physiological demands during the development of the central nervous system

328 (Amlaner et al., 2009) and sex differences in sleep tied to the influence of sex steroids (Mong \&

329 Cusmano, 2016), with younger individuals sleeping more than older individuals (Knutson, 2014;

330 Ohayon et al., 2004; Olds et al., 2010; Steinmeyer et al., 2010; Stuber et al., 2015) and females

331 sleeping more than males in birds and humans (Lendrem, 1983; Mong \& Cusmano, 2016; Roehrs et

332 al., 2006; Steinmeyer et al., 2010; Stuber et al., 2015). Our surprising results here may be an artefact

333 of the tendency of accelerometer-based sleep monitoring to classify resting wakefulness as sleep

334 (Table S12; Ancoli-Israel et al., 2003; de Souza et al., 2003). Older individuals may rest quietly when

335 waking during the night, thus falsely determined to be asleep according to their accelerometry,

336 whereas younger individuals may be more likely to resume activity upon waking. However, if these

337 findings are not the result of a bias in our sleep recording technique, they may reflect variation in the

338 vulnerability to predation among the age-sex classes in this highly sexually dimorphic species

339 (Cheney et al., 2004), with young and female baboons likely realizing a higher cost of sleep than adult

340 males. Individuals may also differ in their sleep patterns due to their ability to gain access to a high-

341 quality sleep location within the group's sleep site. Our results have demonstrated the importance of

342 location to sleep duration. However, group-mates may differ in their access to preferred sleep

343 locations, particularly if preferred locations are limited. Baboon groups are structured by linear

344 dominance hierarchies that shape the priority of access to resources (Cheney \& Seyfarth, 2008; King

345 et al., 2009; Marshall et al., 2015), and individuals can leverage their affiliative and kinship

346 relationships to obtain resources that they would not be able to access based on social rank alone (Sick

347 et al., 2014). Further research is needed to investigate the extent to which these complex social

348 dynamics influence an individual's ability to obtain a preferred sleep location and, thus, a good

349 night's sleep. 
In addition to highlighting social dynamics as a key driver of sleep patterns in group-living

351

352

353

354

355

356

357

358

359

360

361

362

363

364

365

366

367

368

369

370

371

372

373

374

375

376

377

378

379

380

381

382

383

384

385

386 species, our study provides important insights into selective pressures that may have shaped the evolution of human sleep. The physiological requirements for sleep and the homeostatic mechanisms that ensure this requirement is fulfilled have long been assumed to be the key drivers influencing the way that our sleep has evolved and the characteristics of our sleep today. However, we suggest that the criticality of homeostatic control in shaping our sleep patterns could be an artefact of sleeping in an environment devoid of the ecological and social costs that sleep would have presented our ancestors. Evidence suggests that, like baboons, early hominins were extremely vulnerable to nighttime predation in their dry savannah habitats (Brain, 1983; Treves \& Palmqvist, 2007;

Wrangham \& Carmody, 2010). Hominins likely remained vulnerable to nocturnal predation until they began to manipulate fire, around which they could sleep to reduce the risk of predation (Samson \& Nunn, 2015), and some characteristics of our sleep today may be best explained in light of the vulnerability that sleep imparted on our ancestors. For example, modern humans exhibit decreased sleep quality when sleeping in an unfamiliar environment (Tamaki et al., 2016), similar to the baboons in our study. The lower quality sleep resulting from this aptly named "first night effect" is limited to the first night in a new location (Tamaki et al., 2016), and our findings suggest that the first night effect may be conserved from an environment where this first night would have been accompanied by poor information about risk and thus, a higher likelihood of predation. Early hominins would have also experienced a social opportunity cost of sleep, as they likely slept in groups (Samson \& Nunn, 2015; Willems \& van Schaik, 2017) and would have experienced constraints on the time available to maintain their social network during the day, until developing the advanced cognition that enabled a more efficient use of time (Nunn \& Samson, 2018; Samson \& Nunn, 2015). While our sleep has likely evolved substantially from that of our earliest ancestors, with modern human sleep being extremely short and intense compared to that of other primates (Nunn et al., 2016; Nunn \& Samson, 2018), a full understanding of the way we sleep involves considering not only the physiological benefits of sleep, but also its ecological and social costs in the environment in which it evolved.

\section{Materials and Methods}

\section{Data collection}

We monitored sleep and activity patterns in a group of olive baboons at Mpala Research Centre (MRC), a $200 \mathrm{~km}^{2}$ conservancy located on the Laikipia Plateau in central Kenya. We trapped and anesthetized 26 individuals, which comprised more than $80 \%$ of the adults and subadults in the study group (see Strandburg-Peshkin et al., 2015 for details on capture methodology). Upon capture, we noted the age class and sex of each baboon, as well as whether the baboon was lactating. We fit each individual with a GPS and accelerometry collar that recorded the baboon's GPS location at $1 \mathrm{~Hz}$ sampling interval and continuous tri-axial accelerations at $12 \mathrm{~Hz} /$ axis from 06:00 to18:00. From 18:00 to $06: 00$, the collars recorded a 2.5 -second burst of accelerations at $10 \mathrm{~Hz} / \mathrm{axis}$ at the beginning of 
every minute. The collars were programmed to collect data from August 1, 2012 to September 6, 2012, but due to a programming glitch, several collars stopped collecting data prematurely (Table S1). In total, we collected 483 days of GPS data, and 506 nights of accelerometry data. We also collected high-resolution drone imagery of the group's most commonly used sleep site (see Strandburg-Peshkin et al., 2017 for details).

\section{Sleep Analysis}

We used the accelerometry data to classify sleep behavior by adapting a method presented in van Hees et al. 2018 that was developed for extracting metrics of sleep in humans from wearable accelerometry devices. The process of determining the sleep period, defined as the period from sleep onset to waking, is summarized in Fig. 5.

To uniformize the accelerometry sampling schedule, we down-sampled and interpolated the daytime accelerometry data such that it matched the $10 \mathrm{~Hz}$ bursts of accelerometry collected during the night. We calculated the vectorial dynamic body acceleration (VeDBA) using a 0.7 -second timewindow and generated the log of the average VeDBA for the 2.5-second burst each minute. We then calculated a rolling median of the log VeDBA with a 9-minute window. Following van Hees et al. 2018, continuous periods of at least 30 minutes during which the rolling median of the log VeDBA was less than the $10^{\text {th }}$ percentile of the $\log$ VeDBA multiplied by 1.125 were considered sleep blocks. Any blocks within 45 minutes of each other were merged into sleep periods. If this resulted in more than one sleep period, the longest sleep period in the day, defined as a noon-to-noon period, was considered the sleep period. The beginning and end of the sleep period represents the time of sleep onset and waking, respectively. Of the 506 baboon-nights of accelerometry data, we successfully calculated the sleep period for 491 baboon-nights.

[Figure 5]

Adapting the method developed by van Hees and colleagues (2015), we classified each minute epoch both within and outside of the sleep period as representing either sleep or waking behavior. As above, the $10^{\text {th }}$ percentile of the log VeDBA multiplied by 1.125 served as the classification threshold, and we classified epochs as indicating sleep when the log VeDBA for at least three consecutive epochs was below the log VeDBA threshold value. We classified all other epochs as representing waking behavior. Consistent with previous sleep analyses, we measured total sleep time as the total number of minutes of sleep epochs during the sleep period. We measured sleep efficiency as the total sleep time divided by the duration of the sleep period. We measured sleep time during the day - time spent napping - as the number of minutes of sleep epochs from 07:30 to 17:30, as these times were reliably within the waking period (Fig. 1C), and using standardized times prevented a spurious negative correlation between time spent sleeping during the waking period and total sleep time during the sleep period that would result from the waking period prior to or following short sleep periods having a greater number of potential epochs that could be considered sleep. 
The accelerometer units occasionally failed to collect data according to their programmed sampling schedule. Because insufficient data in a given day would prevent a reliable calculation of the threshold value for the sleep classification and produce variability in the number of potential sleep epochs, we did not include data for total sleep time, sleep onset time, waking time, or napping time (both on the prior day and following day) from noon-to-noon periods missing at least $120(8.3 \%)$ accelerometry bursts, which decreased the number of baboon-nights from 491 to 368 . We further removed data for total sleep time, onset time, and waking time from noon-to-noon periods missing at least 20 consecutive accelerometry bursts, as the determination of the sleep period is sensitive to gaps between consecutive accelerometry bursts, resulting in a final number of 354 sleep periods analyzed. We did not remove data for napping time on these days because measuring napping time did not depend on the determination of the sleep period.

\section{Validation of sleep classification algorithm}

The algorithm from which the sleep classification technique is adapted is well-validated using polysomnography $(\mathrm{C}$-statistic $=0.83-0.86)$ to both classify sleep behavior and determine the sleep period in humans (van Hees et al., 2015, 2018). Although the classification of sleep in non-human primates using devices and algorithms that were validated with polysomnography only in humans has become a common practice in sleep research (Barrett et al., 2009; Brutcher \& Nader, 2013; Reinhardt et al., 2019; Reyes et al., 2021; Samson et al., 2018; Sri Kantha \& Suzuki, 2006; Zhdanova et al., 2002), we returned to the study site in July 2019 to validate the accelerometer-based sleep classification. Because logistical and ethical limitations prevent the use of polysomnography in freeranging, highly dexterous animals, we compared the accelerometer-based sleep classification to direct observations of wakeful and sleeping baboons fit with accelerometer collars for validation, as suggested by Rattenborg and colleagues (2017). Behavioral observations were facilitated by highresolution thermal imagery (FLIR T1020, FLIR Systems Inc., Wilsonville, OR, USA). The validation study determined that our accelerometry-based classification of sleep exhibits a 79.9\% accuracy (Table S12; see Supplemental Information for further details of validation study).

\section{Physical activity}

Using the GPS data, we calculated each individual's daily travel distance. To avoid accumulation of GPS positional error overestimating the actual daily travel distance, we calculated daily travel distance only after discretizing the GPS data to 5-meter resolution (Strandburg-Peshkin et al., 2017). We removed travel distance data on days on which a baboon's GPS collar first began taking fixes later than 07:30 or took its last fix before 17:00. Between these times, the group was often on the move, and thus delayed onset and premature offset of GPS devices that infringed upon this period would likely underestimate travel distances. We further removed one individual's data from the first half of the study due to a temporary collar malorientation that resulted in exaggerated GPS error. 
We also calculated cumulative activity during the day from the accelerometry data. Using the continuous $12 \mathrm{~Hz}$ accelerometry data, we calculated VeDBA from 06:00 to 18:00 using a 0.5 second time window, averaged VeDBA over each minute, and then summed these values to generate a cumulative measure of activity during the day.

Visualization of the GPS data indicated that individuals remained reliably stationary until at least 06:15 every day, and thus we determined the location in which each baboon slept from the median of the first 10 GPS locations that occurred before 06:15. If an individual's GPS collar did not successfully collect 10 locations before $06: 15$, its data on this day were excluded from analyses involving sleep location. This resulted in the removal of 9/483 baboon-days of data. In ArcGIS, drone imagery was used to trace the crowns of distinct sleep trees within the group's main sleep site. We determined that an individual slept in a particular tree if its sleep location was within the traced polygon of that tree crown. Sleep locations that fell outside the crown of a tree, likely reflecting minor error in the GPS location estimates, were assigned to the closest sleeping tree. Only 32/469 sleep locations $(6.8 \%)$ had to be assigned to a sleep tree in this manner. In rare cases where an individual's sleep location was greater than $10 \mathrm{~m}$ from the crown of the closest sleep tree $(5 / 474$ cases $-1.1 \%$ of baboon-days), its data on this day were excluded from analysis.

Analysis of the sleep location data revealed that, over the course of the study, the baboons slept in two distinct sleep sites that were separated by approximately $1.5 \mathrm{~km}$. The group slept at their main sleep site for the first 21 nights of the study, and then spent three nights in a different sleep site after a failed leopard attack on the $21^{\text {st }}$ study night. The group then returned to the main site for the duration of the study. In total, they spent 32/35 (91.4\%) nights at their main site and 3/35 (8.6\%) nights at the alternate sleep site. While the entire group slept in a single tree at the less frequently used sleep site, the group's main sleep site contained 10 trees across which the group slept. We performed a permutation test to investigate whether individuals exhibited consistency in the tree in which they chose to sleep. We calculated the Shannon entropy of each individual's sleep tree usage, and we compared these Shannon entropies to those produced from each of 1000 random exchanges of the locations of individuals on each night. Permuted values provide a null distribution controlling for potential sleep tree usage, as the distribution of individuals across the sleep trees each night from the empirical data was maintained in the permuted data. Shannon entropy is a measure of the uncertainty of a random variable, and is given by the equation:

$$
H(X)=-\sum_{X=x} p(x) \log (p(x))
$$

Thus, a lower Shannon entropy in the empirical data compared to the permuted data in this context would signal sleep tree fidelity, with an individual sleeping more often in certain trees and less often in other trees than expected by chance. To determine whether the baboons exhibited significant sleep tree fidelity, we compared the distribution of the group's empirical entropies to the distribution of 
entropies produced from the permutations with a one-tailed two-sample Kolmogorov-Smirnov test. As determining fidelity requires several nights of data, we did not include entropy values, either empirical or permuted, from individuals with less than four nights of data. We also limited this analysis of tree fidelity to the first 15 days of data, as the number of individuals on which we have data decreases sharply after this day (Table S1), which decreases the possible permutations.

After determining that individuals showed non-random sleep tree selection (see Results), we then calculated an individual-specific fidelity index for each tree. This fidelity index was measured as the average number of nights an individual slept in a particular tree in the 1000 permutations subtracted from the number of nights the individual actually slept in that particular tree. Again, we did not calculate fidelity indices for individuals with less than four nights of data.

\section{Pattern of sleep-wake behavior across the group}

We tested whether individuals staggered their periods of nocturnal wakefulness or, conversely, synchronized them beyond the level expected by chance. For this analysis, we subset the data to times between 21:00 and 05:00, as these times consistently fell within the bounds of the sleep period of all individuals. We calculated the proportion of minute epochs across all nights in which at least one group member was awake and the proportion of the group that was synchronized in their behavior (either sleep or wakefulness) during each minute epoch, averaging across all epochs. We then calculated these same proportions, but after applying a random time shift to each individual's time series of sleep-wake epochs on each night (Fig. S9). We repeated this procedure 1000 times to develop a null distribution of the proportion of epochs during the night in which at least one individual is awake and a null distribution of the average proportion of the group that was synchronized, and we compared the empirical proportions to their respective null distributions statistically with a Fisher's exact test. The p-value thus represents the proportion of time-shifted values that were as extreme or more extreme than the empirical value. Shifting the data in time rather than permuting it allowed us to develop null distributions while maintaining the autocorrelation structure of the data.

To confirm the robustness of our findings, we again tested for collective vigilance and synchronization, comparing the empirical values defined above to null distributions produced using an alternative method. In this method, rather than applying a random time shift to each night of each individual's data, we maintained the real time associated with the time series data, but we permuted the night associated with each time series (Fig. S10). We compared empirical values to the null distributions created by these night permutations with a Fisher's exact test.

\section{Statistical analysis of sleep}

Data were processed using the statistical analysis software R version 4.0.5 (R Core Team, 2021). We only included the first 20 study nights in the analyses of sleep, except where specified, due to concerns that the leopard attack that occurred on the $21^{\text {st }}$ night could potentially disrupt typical sleep patterns. To compare the effects of various physiological, ecological, and social predictors of 
sleep, we modeled total sleep time with a Bayesian linear mixed model (LMM), with random effects of individual identity and night, and fixed effects of age, sex, distance traveled in the preceding day, napping time during the preceding day, relative time spent sleeping the previous night, the identity of the sleep tree, fidelity index for the current sleep tree, the relative number of individuals in the sleep tree, the phase of the moon, and minimum ambient temperature during the night. We created a separate model that included cumulative VeDBA instead of distance travelled because cumulative daytime VeDBA was highly correlated with distance travelled during the day. An individual's relative time spent sleeping the previous night was measured as the difference between its total sleep time on the previous night and its average total sleep time. This relative measure controlled for positive correlations between total sleep time on previous night and current night total sleep time that would result purely from among-individual variation in total sleep time - a scenario that would not be sufficiently controlled for by the individual identity random effect in this model. We calculated the relative number of individuals in the sleep tree by dividing the number of individuals in the sleep tree by the total number of individuals who were successfully assigned to a sleep tree on that given night, to control for the decrease in the number of individuals in each sleep tree over the course of the study that resulted from premature termination of data collection in several collars. Moon phase was a continuous variable realizing values from 0 to 1 (with 0 representing a new moon, and 1 representing a full moon), and we collected this data for the days of the study using the sunCalc package in $\mathrm{R}$ (Benoit \& Elmarhraoui, 2019). The minimum ambient temperature represented the minimum temperature at the sleep site during the night, determined using interpolated ECMWF air temperature ( $2 \mathrm{~m}$ above ground) data obtained with the Env-DATA functionality (Dodge et al., 2013) provided on Movebank data repository (www.movebank.org). We standardized all response and predictor variables to allow for comparison of effect sizes among variables. To increase the interpretability of the total sleep time model, we reran the model without standardized variables. Effect sizes reported in the main text are derived from the standardized model, whereas figures produced in the main text, as well as the interpretation of the effect of each variable on the unstandardized sleep time are derived from the model with unstandardized variables.

To examine the effect of the leopard attack and subsequent sleep site change on sleep parameters, we modeled the effect of particular nights on sleep parameters with a Bayesian LMM. Specifically, we divided data into the following categories: all nights before the leopard attack, the night of the leopard attack, the first night in the new sleep site (i.e. the night following the leopard attack), the second night in the new sleep site, the third night in the new sleep site, and the remainder of study nights, during which the group slept in its main sleep site. Aside from this categorical night variable, we also included age, sex, distance traveled in the preceding day, napping time during the preceding day, relative time spent sleeping the previous night, the phase of the moon, and minimum ambient temperature as fixed effects in the models with random intercepts for individual identity. In 
these models, we did not include sleep tree identity, number of individuals in the sleep tree, and sleep tree fidelity score, as the entire group slept in a single tree in the less commonly used sleep site.

We further tested for the effect of prior sleep debt on sleep behavior by modeling the effect of total sleep time on time spent napping the following day. We modeled this relationship with a Bayesian LMM, using individual identity and day as random intercepts. We also assessed how the likelihood of sleep progressed through the night. We used a generalized additive mixed model (GAMM) to model the log-odds of a baboon being asleep in a given epoch as a function of the duration of that epoch from the beginning of the sleep period, scaled such that 0 represents the beginning of the sleep period and 1 represents the end of the sleep period. We included individual identity and night as random intercepts, and to account for autocorrelation in the response variable, we also included an AR1 term in the model.

Lastly, we tested whether individuals showed higher synchronization of their sleep-wake patterns when sharing the same sleep tree than when inhabiting different trees. With a Bayesian LMM, we modeled the synchronization score between dyads on each night, calculated as the number of minutes from 21:00 to 05:00 in which members of the dyad exhibited the same behavior divided by the total number minutes in which both individuals had data. We included a binary predictor variable indicating whether dyad members were in the same tree as the only fixed effect variable, and night, the identity of both individuals in the dyad, as well as the identity of the dyad as random intercept variables.

We carried out all Bayesian analyses with the "brms" package in R (Bürkner, 2017). We used diffuse, mean-zero Gaussian priors for all predictor variables. Model estimates are based off of four independent Hamiltonian Monte Carlo chains with 5000 iterations, 2500 of which were burn-in iterations. Trace plots indicated that mixing was sufficient and that the four chains converged on the same posterior region. Model estimates reported in the text represent the mean of the posterior distribution, along with the lower and upper 95\% credible interval bounds from the standardized models. We used package "mgcv" in R (Wood, 2011) to fit the GAMM to the sleep epoch data, using a thin plate spline smoothing term with 10 knots.

\section{Acknowledgments}

We are grateful to the Kenya National Science and Technology Council (NCTS/RCD/12B/012/26B, NACOSTI/P/15/5727/4608, NACOSTI/P/19/55517/24299, NACOSTI/P/20/1407), Kenyan Wildlife Service (KWS/BRM/5001), and Mpala Research Centre for permission to conduct research. Research procedures received IACUC approval (Smithsonian Tropical Research Institute, assurance number 2012.0601.2015; University of California, Davis, assurance number 20442). We thank Ariana Strandburg-Peshkin, Alison Ashbury, and Niels Rattenborg for helpful comments on previous drafts of the manuscript. We thank M Wikelski, E Bermingham, D Rubenstein, M Kinnaird, D Carlino, and B Tripard for logistical support, as well as R Kays, S Murray, M Mutinda, R Lessnau, S Alavi, J Nairobi, F Kuemmeth, and W Heidrich for field 
606

607

608

609

610

611

612

613

614

615

616

617

618

619

620

621

622

623

624

625

626

627

628

629

630

631

632

633

634

635

636

637

638

639

assistance. This research received funding from the Max Planck Institute for Ornithology, the Smithsonian Tropical Research Institute, and University of California, Davis. RH and MCC were supported by the National Science Foundation (IIS $1514174 \&$ IOS 1250895). MCC received additional support from a Packard Foundation Fellowship (2016-65130), and the Alexander von Humboldt Foundation in the framework of the Alexander von Humboldt Professorship endowed by the Federal Ministry of Education and Research. JCL was supported by a National Science Foundation Graduate Research Fellowship, the National Science Foundation Graduate Research Internship Program, a Dean's Distinguished Graduate Fellowship from the University of California, Davis, and a Richard G. Coss Wildlife Research Fellowship. Further support for the project was also provided by the Center for the Advanced Study of Collective Behavior at the University of Konstanz, Deutsche Forschungsgemeinschaft Centre of Excellence 2117 (ID: 422037984$).$

\section{Author Contributions}

J.C.L., R.H., and M.C.C. conceived the project. M.C.C. oversaw the data collection. J.C.L., R.H., and C.L.N analyzed the data and prepared the figures. J.C.L. led the writing of the manuscript, to which all authors contributed.

\section{Competing interests}

The authors declare no competing interests.

\section{Data availability}

GPS and accelerometry data generated during this study are published and available in the Movebank repository (www.movebank.org; Crofoot et al. 2021). Drone imagery is publicly available for download from Dryad (http://dx.doi.org/10.5061/dryad.6h5b7). Accelerometry data and behavioral scoring data from the 2019 sleep validation study is also publicly available for download from Dryad (https://doi.org/10.5061/dryad.p5hqbzkqf).

\section{References}

Altmann, S. A., \& Altmann, J. (1970). Baboon ecology: African field research. University of Chicago Press, Chicago.

Amlaner, C. J., Phil, D., \& Fuller, P. M. (2009). Basics of sleep guide. Sleep Research Society.

Ancoli-Israel, S., Cole, R., Alessi, C., Chambers, M., Moorcroft, W., \& Pollak, C. P. (2003). The Role of Actigraphy in the Study of Sleep and Circadian Rhythms. Sleep, 26(3), 342-392. https://doi.org/10.1093/sleep/26.3.342

Aulsebrook, A. E., Jones, T. M., Rattenborg, N. C., Roth, T. C., \& Lesku, J. A. (2016). Sleep Ecophysiology: Integrating Neuroscience and Ecology. Trends in Ecology \& Evolution, 31(8), 590-599. https://doi.org/10.1016/j.tree.2016.05.004 
640 Bäckman, J., Andersson, A., Pedersen, L., Sjöberg, S., Tøttrup, A. P., \& Alerstam, T. (2017).

641

642

643

644

645

646

647

648

649

650

651

652

653

654

656

657

658

659

660

661

662

663

664

665

666

667 Actogram analysis of free-flying migratory birds: New perspectives based on acceleration logging. Journal of Comparative Physiology A, 203(6-7), 543-564. https://doi.org/10.1007/s00359-017-1165-9

Barrett, C. E., Noble, P., Hanson, E., Pine, D. S., Winslow, J. T., \& Nelson, E. E. (2009). Early adverse rearing experiences alter sleep-wake patterns and plasma cortisol levels in juvenile rhesus monkeys. Psychoneuroendocrinology, 34(7), 1029-1040. https://doi.org/10.1016/j.psyneuen.2009.02.002

Basner, M., Rao, H., Goel, N., \& Dinges, D. F. (2013). Sleep deprivation and neurobehavioral dynamics. Current Opinion in Neurobiology, 23(5), 854-863. https://doi.org/10.1016/j.conb.2013.02.008

Benoit, T., \& Elmarhraoui, A. (2019). suncalc: Compute Sun Position, Sunlight Phases, Moon Position and Lunar Phase (R package version 0.5.0) [Computer software]. https://CRAN.Rproject.org/package $=$ suncalc

Brain, C. K. (1983). The hunters or the hunted?: An introduction to African cave taphonomy. University of Chicago Press.

Brutcher, R. E., \& Nader, M. A. (2013). The relationship between cocaine self-administration and actigraphy-based measures of sleep in adult rhesus monkeys. Psychopharmacology, 229(2), 267-274. https://doi.org/10.1007/s00213-013-3101-1

Bürkner, P.-C. (2017). brms: An R Package for Bayesian Multilevel Models Using Stan. Journal of Statistical Software, 80(1), 1-28. https://doi.org/10.18637/jss.v080.i01

Busse, C. (1980). Leopard and lion predation upon chacma baboons living in the Moremi Wildlife Reserve. Botswana Notes and Records, 15-21.

Cameron, E. Z., Setsaas, T. H., \& Linklater, W. L. (2009). Social bonds between unrelated females increase reproductive success in feral horses. Proceedings of the National Academy of Sciences, 106(33), 13850-13853. https://doi.org/10.1073/pnas.0900639106

Campos, F. A., Villavicencio, F., Archie, E. A., Colchero, F., \& Alberts, S. C. (2020). Social bonds, social status and survival in wild baboons: A tale of two sexes. Philosophical Transactions of 

https://doi.org/10.1098/rstb.2019.0621

Cheney, D. L., \& Seyfarth, R. M. (2008). Baboon metaphysics: The evolution of a social mind. University of Chicago Press.

Cheney, D. L., Seyfarth, R. M., Fischer, J., Beehner, J., Bergman, T., Johnson, S. E., Kitchen, D. M., Palombit, R. A., Rendall, D., \& Silk, J. B. (2004). Factors affecting reproduction and mortality among baboons in the Okavango Delta, Botswana. International Journal of Primatology, 25(2), 401-428.

Chowdhury, B., \& Shafer, O. T. (2020). Why the young sleep longer. ELife, 9, e56833. https://doi.org/10.7554/eLife.56833

Cirelli, C., \& Tononi, G. (2008). Is Sleep Essential? PLOS Biology, 6(8), e216. https://doi.org/10.1371/journal.pbio.0060216

Cowlishaw, G. (1994). Vulnerability To Predation in Baboon Populations. Behaviour, 131(3-4), 293304. https://doi.org/10.1163/156853994X00488

Davimes, J. G., Alagaili, A. N., Bhagwandin, A., Bertelsen, M. F., Mohammed, O. B., Bennett, N. C.,

de Souza, L., Benedito-Silva, A. A., Pires, M. L. N., Poyares, D., Tufik, S., \& Calil, H. M. (2003). Further Validation of Actigraphy for Sleep Studies. Sleep, 26(1), 81-85. https://doi.org/10.1093/sleep/26.1.81

Dement, W. (1960). The Effect of Dream Deprivation. Science, 131(3415), 1705-1707.

Dodge, S., Bohrer, G., Weinzierl, R., Davidson, S. C., Kays, R., Douglas, D., Cruz, S., Han, J., Brandes, D., \& Wikelski, M. (2013). The environmental-data automated track annotation 1(1), 3. https://doi.org/10.1186/2051-3933-1-3

Dunbar, R. I. (1992). Time: A hidden constraint on the behavioural ecology of baboons. Behavioral Ecology and Sociobiology, 31(1), 35-49. 
Forrester, T. D., Casady, D. S., \& Wittmer, H. U. (2015). Home sweet home: Fitness consequences of site familiarity in female black-tailed deer. Behavioral Ecology and Sociobiology, 69(4), 603 612. https://doi.org/10.1007/s00265-014-1871-z

Frère, C. H., Krützen, M., Mann, J., Connor, R. C., Bejder, L., \& Sherwin, W. B. (2010). Social and genetic interactions drive fitness variation in a free-living dolphin population. Proceedings of the National Academy of Sciences, 107(46), 19949-19954. https://doi.org/10.1073/pnas.1007997107

Gangwisch, J. E. (2014). A Review of Evidence for the Link Between Sleep Duration and Hypertension. American Journal of Hypertension, 27(10), 1235-1242. https://doi.org/10.1093/ajh/hpu071

Gehr, B., Bonnot, N. C., Heurich, M., Cagnacci, F., Ciuti, S., Hewison, A. M., Gaillard, J.-M., Ranc, N., Premier, J., \& Vogt, K. (2020). Stay home, stay safe—Site familiarity reduces predation risk in a large herbivore in two contrasting study sites. Journal of Animal Ecology, 89(6), $1329-1339$.

Gravett, N., Bhagwandin, A., Sutcliffe, R., Landen, K., Chase, M. J., Lyamin, O. I., Siegel, J. M., \& Manger, P. R. (2017). Inactivity/sleep in two wild free-roaming African elephant matriarchs Does large body size make elephants the shortest mammalian sleepers? PLOS ONE, 12(3), e0171903. https://doi.org/10.1371/journal.pone.0171903

Hayward, M. W., Henschel, P., O’Brien, J., Hofmeyr, M., Balme, G., \& Kerley, G. I. H. (2006). Prey preferences of the leopard (Panthera pardus). Journal of Zoology, 270(2), 298-313. https://doi.org/10.1111/j.1469-7998.2006.00139.x

Hoffmann, K., Coolen, A., Schlumbohm, C., Meerlo, P., \& Fuchs, E. (2012). Remote long-term registrations of sleep-wake rhythms, core body temperature and activity in marmoset monkeys. Behavioural Brain Research, 235(2), 113-123. https://doi.org/10.1016/j.bbr.2012.07.033

Isbell, L. A., Bidner, L. R., Van Cleave, E. K., Matsumoto-Oda, A., \& Crofoot, M. C. (2018). GPSidentified vulnerabilities of savannah-woodland primates to leopard predation and their 
implications for early hominins. Journal of Human Evolution, 118, 1-13. https://doi.org/10.1016/j.jhevol.2018.02.003

Jones, S. E., van Hees, V. T., Mazzotti, D. R., Marques-Vidal, P., Sabia, S., van der Spek, A., Dashti, H. S., Engmann, J., Kocevska, D., Tyrrell, J., Beaumont, R. N., Hillsdon, M., Ruth, K. S., Tuke, M. A., Yaghootkar, H., Sharp, S. A., Ji, Y., Harrison, J. W., Freathy, R. M., ... Wood, A. R. (2019). Genetic studies of accelerometer-based sleep measures yield new insights into human sleep behaviour. Nature Communications, 10(1), 1585. https://doi.org/10.1038/s41467-019-09576-1

Kanady, J. C., Drummond, S. P. A., \& Mednick, S. C. (2011). Actigraphic assessment of a

Karamihalev, S., Flachskamm, C., Eren, N., Kimura, M., \& Chen, A. (2019). Social context and dominance status contribute to sleep patterns and quality in groups of freely-moving mice.

King, A. J., Isaac, N. J., \& Cowlishaw, G. (2009). Ecological, social, and reproductive factors shape producer-scrounger dynamics in baboons. Behavioral Ecology, 20(5), 1039-1049.

Kitamura, S., Katayose, Y., Nakazaki, K., Motomura, Y., Oba, K., Katsunuma, R., Terasawa, Y., Enomoto, M., Moriguchi, Y., Hida, A., \& Mishima, K. (2016). Estimating individual optimal sleep duration and potential sleep debt. Scientific Reports, 6(1), 35812. https://doi.org/10.1038/srep35812

Knutson, K. L. (2014). Sleep duration, quality, and timing and their associations with age in a

Ladha, C., \& Hoffman, C. L. (2018). A Combined Approach to Predicting Rest in Dogs Using Accelerometers. Sensors, 18(8), 2649. https://doi.org/10.3390/s18082649 life in the face of danger. Behavioral Ecology and Sociobiology, 70(4), 449-458. https://doi.org/10.1007/s00265-016-2075-5 
Lendrem, D. W. (1983). Sleeping and vigilance in birds. I. Field observations of the mallard (Anas platyrhynchos). Animal Behaviour. https://agris.fao.org/agrissearch/search.do?recordID=US201302605924

Lesku, J. A., Meyer, L. C. R., Fuller, A., Maloney, S. K., Dell’Omo, G., Vyssotski, A. L., \& Rattenborg, N. C. (2011). Ostriches Sleep like Platypuses. PLOS ONE, 6(8), e23203. https://doi.org/10.1371/journal.pone.0023203

Lesku, J. A., Rattenborg, N. C., Valcu, M., Vyssotski, A. L., Kuhn, S., Kuemmeth, F., Heidrich, W., \& Kempenaers, B. (2012). Adaptive Sleep Loss in Polygynous Pectoral Sandpipers. Science, 337(6102), 1654-1658. https://doi.org/10.1126/science.1220939

Lima, S. L., Rattenborg, N. C., Lesku, J. A., \& Amlaner, C. J. (2005). Sleeping under the risk of predation. Animal Behaviour, 70(4), 723-736. https://doi.org/10.1016/j.anbehav.2005.01.008

Lin, B., Foxfoot, I. R., Miller, C. M., Venkatamaran, V. V., Kerby, J. T., Bechtold, E. K., Kellogg, B. S., Nguyen, N., \& Fashing, P. J. (2020). Leopard predation on gelada monkeys at Guassa, Ethiopia. American Journal of Primatology, 82(2), e23098. https://doi.org/10.1002/ajp.23098

Lyamin, O. I., Kosenko, P. O., Korneva, S. M., Vyssotski, A. L., Mukhametov, L. M., \& Siegel, J. M. (2018). Fur Seals Suppress REM Sleep for Very Long Periods without Subsequent Rebound. Current Biology, 28(12), 2000-2005.e2. https://doi.org/10.1016/j.cub.2018.05.022

Malungo, I. B., Gravett, N., Bhagwandin, A., Davimes, J. G., \& Manger, P. R. (2021). Sleep in two free-roaming blue wildebeest (Connochaetes taurinus), with observations on the agreement of polysomnographic and actigraphic techniques. IBRO Neuroscience Reports, 10, 142-152. https://doi.org/10.1016/j.ibneur.2021.02.005

Marshall, H. H., Carter, A. J., Ashford, A., Rowcliffe, J. M., \& Cowlishaw, G. (2015). Social effects on foraging behavior and success depend on local environmental conditions. Ecology and Evolution, 5(2), 475-492.

Miller, P. J. O., Aoki, K., Rendell, L. E., \& Amano, M. (2008). Stereotypical resting behavior of the sperm whale. Current Biology, 18(1), R21-R23. https://doi.org/10.1016/j.cub.2007.11.003 
Mitani, Y., Andrews, R. D., Sato, K., Kato, A., Naito, Y., \& Costa, D. P. (2010). Three-dimensional resting behaviour of northern elephant seals: Drifting like a falling leaf. Biology Letters, 6(2), 163-166. https://doi.org/10.1098/rsbl.2009.0719

Mong, J. A., \& Cusmano, D. M. (2016). Sex differences in sleep: Impact of biological sex and sex steroids. Philosophical Transactions of the Royal Society B: Biological Sciences, 371(1688), 20150110. https://doi.org/10.1098/rstb.2015.0110

Nunn, C. L., \& Samson, D. R. (2018). Sleep in a comparative context: Investigating how human sleep differs from sleep in other primates. American Journal of Physical Anthropology, 166(3), 601-612. https://doi.org/10.1002/ajpa.23427

Nunn, C. L., Samson, D. R., \& Krystal, A. D. (2016). Shining evolutionary light on human sleep and sleep disorders. Evolution, Medicine, and Public Health, 2016(1), 227-243. https://doi.org/10.1093/emph/eow018

Ohayon, M. M., Carskadon, M. A., Guilleminault, C., \& Vitiello, M. V. (2004). Meta-Analysis of Quantitative Sleep Parameters From Childhood to Old Age in Healthy Individuals: Developing Normative Sleep Values Across the Human Lifespan. Sleep, 27(7), 1255-1273. https://doi.org/10.1093/sleep/27.7.1255

Olds, T., Blunden, S., Petkov, J., \& Forchino, F. (2010). The relationships between sex, age, geography and time in bed in adolescents: A meta-analysis of data from 23 countries. Sleep Medicine Reviews, 14(6), 371-378. https://doi.org/10.1016/j.smrv.2009.12.002

Patel, S. I., Miller, B. W., Kosiorek, H. E., Parish, J. M., Lyng, P. J., \& Krahn, L. E. (2017). The Effect of Dogs on Human Sleep in the Home Sleep Environment. Mayo Clinic Proceedings, 92(9), 1368-1372. https://doi.org/10.1016/j.mayocp.2017.06.014

Qasem, L., Cardew, A., Wilson, A., Griffiths, I., Halsey, L. G., Shepard, E. L., Gleiss, A. C., \& Wilson, R. (2012). Tri-axial dynamic acceleration as a proxy for animal energy expenditure; should we be summing values or calculating the vector? PloS One, 7(2), e31187.

Qin, D.-D., Feng, S.-F., Zhang, F.-Y., Wang, N., Sun, W.-J., Zhou, Y., Xiong, T.-F., Xu, X.-L., Yang, X.-T., Zhang, X., Zhu, X., Hu, X.-T., Xiong, L., Liu, Y., \& Chen, Y.-C. (2020). Potential use of actigraphy to measure sleep in monkeys: Comparison with behavioral analysis from 
videography. Zoological Research, 41(4), 437-443. https://doi.org/10.24272/j.issn.20958137.2020 .056

R Core Team. (2021). R: A language and environment for statistical computing. R Foundation for Statistical Computing (4.0.5) [Computer software]. R Foundation for Statistical Computing. https://www.R-project.org/

Rattenborg, N. C., de la Iglesia, H. O., Kempenaers, B., Lesku, J. A., Meerlo, P., \& Scriba, M. F. (2017). Sleep research goes wild: New methods and approaches to investigate the ecology, evolution and functions of sleep. Philosophical Transactions of the Royal Society B: Biological Sciences, 372(1734), 20160251.

Rattenborg, N. C., Voirin, B., Cruz, S. M., Tisdale, R., Dell’Omo, G., Lipp, H.-P., Wikelski, M., \& Vyssotski, A. L. (2016). Evidence that birds sleep in mid-flight. Nature Communications, 7(1), 12468. https://doi.org/10.1038/ncomms 12468

Rattenborg, N. C., Voirin, B., Vyssotski, A. L., Kays, R. W., Spoelstra, K., Kuemmeth, F., Heidrich,

Rechtschaffen, A., \& Bergmann, B. M. (2002). Sleep deprivation in the rat: An update of the 1989 paper. Sleep, 25(1), 18-24. https://doi.org/10.1093/sleep/25.1.18

Reinhardt, K. D. (2020). Wild primate sleep: Understanding sleep in an ecological context. Current Opinion in Physiology, 15, 238-244. https://doi.org/10.1016/j.cophys.2020.04.002 (2019). Environment shapes sleep patterns in a wild nocturnal primate. Scientific Reports, 9(1), 9939. https://doi.org/10.1038/s41598-019-45852-2 influence of lunar phase and meteorological variables on activity in Hylobates moloch and Hylobates pileatus. Primates, 62(5), 749-759. https://doi.org/10.1007/s10329-021-00920-y 
Riehl, C., \& Strong, M. J. (2018). Stable social relationships between unrelated females increase individual fitness in a cooperative bird. Proceedings of the Royal Society B: Biological Sciences, 285(1876), 20180130. https://doi.org/10.1098/rspb.2018.0130

Roehrs, T., Kapke, A., Roth, T., \& Breslau, N. (2006). Sex differences in the polysomnographic sleep of young adults: A community-based study. Sleep Medicine, 7(1), 49-53. https://doi.org/10.1016/j.sleep.2005.05.008

Roth, T. C., Rattenborg, N. C., \& Pravosudov, V. V. (2010). The ecological relevance of sleep: The trade-off between sleep, memory and energy conservation. Philosophical Transactions of the Royal Society B: Biological Sciences, 365(1542), 945-959. https://doi.org/10.1098/rstb.2009.0209

Samson, D. R., Bray, J., \& Nunn, C. L. (2018). The cost of deep sleep: Environmental influences on sleep regulation are greater for diurnal lemurs. American Journal of Physical Anthropology, 166(3), 578-589. https://doi.org/10.1002/ajpa.23455

Samson, D. R., Crittenden, A. N., Mabulla, I. A., Mabulla, A. Z. P., \& Nunn, C. L. (2017). Chronotype variation drives night-time sentinel-like behaviour in hunter-gatherers. Proceedings of the Royal Society B: Biological Sciences, 284(1858), 20170967. https://doi.org/10.1098/rspb.2017.0967

Samson, D. R., \& Nunn, C. L. (2015). Sleep intensity and the evolution of human cognition. Evolutionary Anthropology: Issues, News, and Reviews, 24(6), 225-237. https://doi.org/10.1002/evan.21464

Scriba, M. F., Ducrest, A.-L., Henry, I., Vyssotski, A. L., Rattenborg, N. C., \& Roulin, A. (2013). Linking melanism to brain development: Expression of a melanism-related gene in barn owl feather follicles covaries with sleep ontogeny. Frontiers in Zoology, 10(1), 42. https://doi.org/10.1186/1742-9994-10-42

Sellers, W. I., \& Crompton, R. H. (2004). Automatic Monitoring of Primate Locomotor Behaviour Using Accelerometers. Folia Primatologica, 75(4), 279-293. https://doi.org/10.1159/000078939 
Shambroom, J. R., Fábregas, S. E., \& Johnstone, J. (2012). Validation of an automated wireless system to monitor sleep in healthy adults. Journal of Sleep Research, 21(2), 221-230. https://doi.org/10.1111/j.1365-2869.2011.00944.x

Shiromani, P. J., Lu, J., Wagner, D., Thakkar, J., Greco, M. A., Basheer, R., \& Thakkar, M. (2000). Compensatory sleep response to $12 \mathrm{~h}$ wakefulness in young and old rats. American Journal of Physiology-Regulatory, Integrative and Comparative Physiology, 278(1), R125-R133. https://doi.org/10.1152/ajpregu.2000.278.1.R125

Sick, C., Carter, A. J., Marshall, H. H., Knapp, L. A., Dabelsteen, T., \& Cowlishaw, G. (2014). Evidence for varying social strategies across the day in chacma baboons. Biology Letters, 10(7), 20140249.

Siegel, J. M. (2008). Do all animals sleep? Trends in Neurosciences, 31(4), 208-213.

Silk, J. B., Beehner, J. C., Bergman, T. J., Crockford, C., Engh, A. L., Moscovice, L. R., Wittig, R. M., Seyfarth, R. M., \& Cheney, D. L. (2009). The benefits of social capital: Close social bonds among female baboons enhance offspring survival. Proceedings of the Royal Society B: Biological Sciences, 276(1670), 3099-3104. https://doi.org/10.1098/rspb.2009.0681

Snyder, F. (1966). Toward an evolutionary theory of dreaming. American Journal of Psychiatry, 123, $121-136$.

Sri Kantha, S., \& Suzuki, J. (2006). Sleep quantitation in common marmoset, cotton top tamarin and squirrel monkey by non-invasive actigraphy. Comparative Biochemistry and Physiology Part A: Molecular \& Integrative Physiology, 144(2), 203-210. https://doi.org/10.1016/j.cbpa.2006.02.043

Steinmeyer, C., Schielzeth, H., Mueller, J. C., \& Kempenaers, B. (2010). Variation in sleep behaviour in free-living blue tits, Cyanistes caeruleus: Effects of sex, age and environment. Animal Behaviour, 80(5), 853-864. https://doi.org/10.1016/j.anbehav.2010.08.005

Stickgold, R. (2005). Sleep-dependent memory consolidation. Nature, 437(7063), 1272-1278.

Strandburg-Peshkin, A., Farine, D. R., Couzin, I. D., \& Crofoot, M. C. (2015). Shared decisionmaking drives collective movement in wild baboons. Science, 348(6241), 1358-1361. 
Strandburg-Peshkin, A., Farine, D. R., Crofoot, M. C., \& Couzin, I. D. (2017). Habitat and social factors shape individual decisions and emergent group structure during baboon collective movement. ELife, 6, e19505. https://doi.org/10.7554/eLife.19505

Stuber, E. F., Dingemanse, N. J., Kempenaers, B., \& Mueller, J. C. (2015). Sources of intraspecific variation in sleep behaviour of wild great tits. Animal Behaviour, 106, 201-221. https://doi.org/10.1016/j.anbehav.2015.05.025

Suzuki, M., Nakayama, M., Ando, K. B., Arima, S., Nakamura, Y., Yokota, M., \& Murakami, S. (2018). Sleep Disturbance and Hyperactivity Detected by Actigraphy in Rats with Allergic Rhinitis or Attention-Deficit Hyperactivity Disorder. The Tohoku Journal of Experimental Medicine, 246(2), 65-71. https://doi.org/10.1620/tjem.246.65

Tamaki, M., Bang, J. W., Watanabe, T., \& Sasaki, Y. (2016). Night Watch in One Brain Hemisphere during Sleep Associated with the First-Night Effect in Humans. Current Biology, 26(9), 1190-1194. https://doi.org/10.1016/j.cub.2016.02.063

Treves, A., \& Palmqvist, P. (2007). Reconstructing Hominin Interactions with Mammalian Carnivores (6.0-1.8 Ma). In S. L. Gursky \& K. A. I. Nekaris (Eds.), Primate Anti-Predator Strategies (pp. 355-381). Springer US. https://doi.org/10.1007/978-0-387-34810-0_17

van Hees, V. T., Sabia, S., Anderson, K. N., Denton, S. J., Oliver, J., Catt, M., Abell, J. G., Kivimäki, M., Trenell, M. I., \& Singh-Manoux, A. (2015). A novel, open access method to assess sleep duration using a wrist-worn accelerometer. PloS One, 10(11), e0142533.

van Hees, V. T., Sabia, S., Jones, S. E., Wood, A. R., Anderson, K. N., Kivimäki, M., Frayling, T. M., Pack, A. I., Bucan, M., Trenell, M. I., Mazzotti, D. R., Gehrman, P. R., Singh-Manoux, B. A., \& Weedon, M. N. (2018). Estimating sleep parameters using an accelerometer without sleep diary. Scientific Reports, 8(1), 12975. https://doi.org/10.1038/s41598-018-31266-z

Voirin, B., Scriba, M. F., Martinez-Gonzalez, D., Vyssotski, A. L., Wikelski, M., \& Rattenborg, N. C. (2014). Ecology and Neurophysiology of Sleep in Two Wild Sloth Species. Sleep, 37(4), 753-761. https://doi.org/10.5665/sleep.3584

Vyazovskiy, V. V. (2015). Sleep, recovery, and metaregulation: Explaining the benefits of sleep. Nature and Science of Sleep, 7, 171-184. https://doi.org/10.2147/NSS.S54036 
913 Ward, A., \& Webster, M. (2016). Sociality: The behaviour of group-living animals.

914 Willems, E. P., \& van Schaik, C. P. (2017). The social organization of Homo ergaster: Inferences

915 from anti-predator responses in extant primates. Journal of Human Evolution, 109, 11-21.

$916 \quad$ https://doi.org/10.1016/j.jhevol.2017.05.003

917 Wood, S. N. (2011). Fast stable restricted maximum likelihood and marginal likelihood estimation of semiparametric generalized linear models. Journal of the Royal Statistical Society: Series B (Statistical Methodology), 73(1), 3-36. https://doi.org/10.1111/j.1467-9868.2010.00749.x

920 Wrangham, R., \& Carmody, R. (2010). Human adaptation to the control of fire. Evolutionary Anthropology: Issues, News, and Reviews, 19(5), 187-199. https://doi.org/10.1002/evan.20275

Wright, A. J., Akamatsu, T., Mouritsen, K. N., Sveegaard, S., Dietz, R., \& Teilmann, J. (2017). Silent porpoise: Potential sleeping behaviour identified in wild harbour porpoises. Animal Behaviour, 133, 211-222. https://doi.org/10.1016/j.anbehav.2017.09.015

Yoder, J. M., Marschall, E. A., \& Swanson, D. A. (2004). The cost of dispersal: Predation as a function of movement and site familiarity in ruffed grouse. Behavioral Ecology, 15(3), 469-

Zhdanova, I. V., Geiger, D. A., Schwagerl, A. L., Leclair, O. U., Killiany, R., Taylor, J. A., Rosene, 


\section{Figures}

944

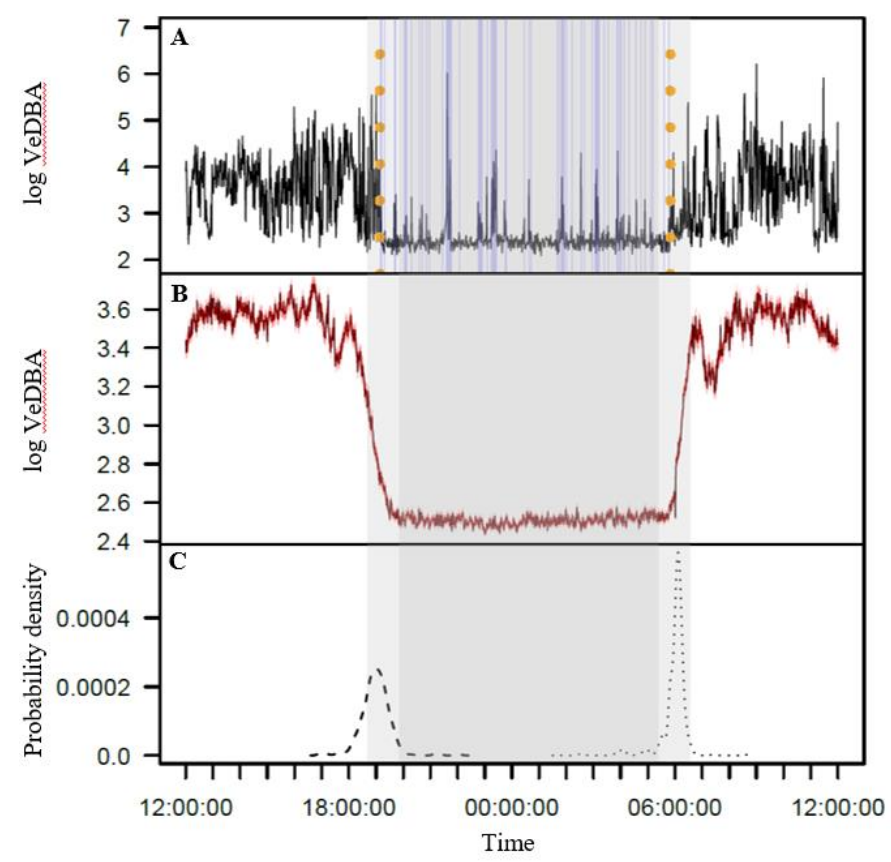

Figure 1. Extracting activity and sleep from accelerometry in a group of wild olive baboons. Adapting algorithms developed by van Hees and colleagues $(2015,2018)$, we used the vectorial dynamic body acceleration (VeDBA), a measure of overall activity, to determine the sleep onset and awakening times (A; orange dotted lines), as well as periods of wake after sleep onset (A; blue shading) for each individual baboon on each day. These metrics allowed us to calculate the total sleep time, sleep period duration, and sleep efficiency as well. The plot (A) shows the data of one individual within a single noon-to-noon period as an example. Averaged across all individuals on all nights ( $N=354$ baboons-nights), the log VeDBA shows that baboons exhibit activity patterns typical of a diurnal animal with monophasic sleep (B), with a consolidated period of very low levels of activity during the night. Although the timing of waking (C; dotted line) was more consistent across the group and across the study period than the timing of sleep onset (C; dashed line), both sleep onset and waking typically occurred within astronomical twilight. The red shading in (B) indicates $\pm 1 \mathrm{SE}$. In all subplots, the grey shaded region depicts the period between sunset and sunrise, with double shading from the end of astronomical twilight to the beginning of morning astronomical twilight. 

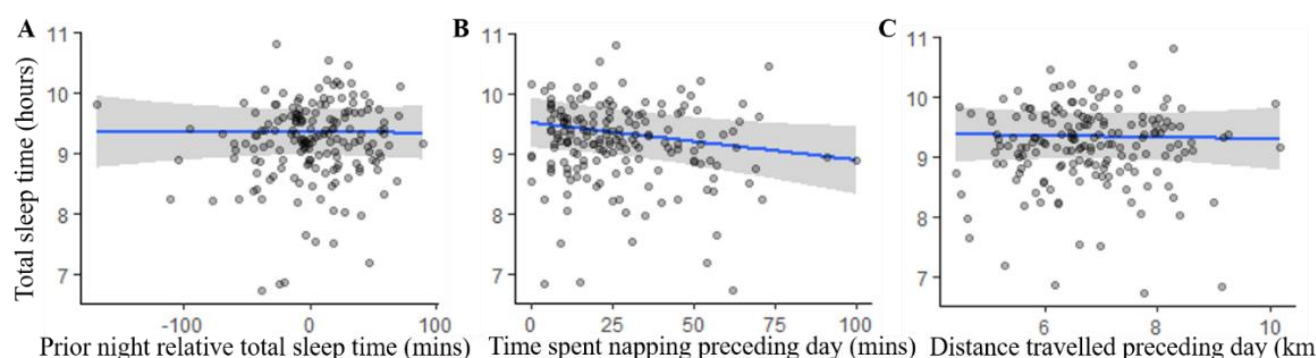

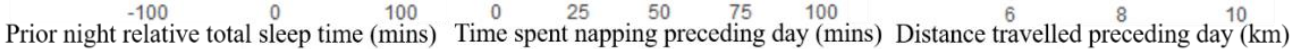

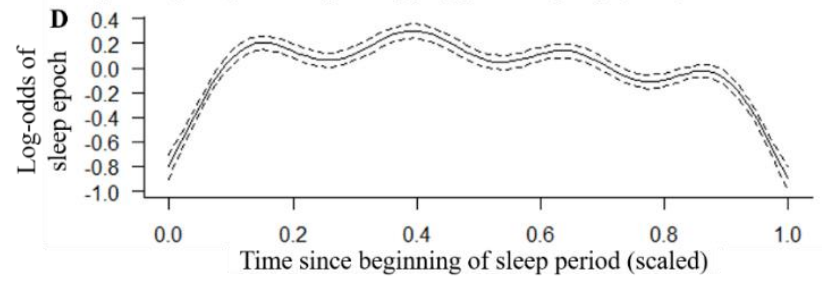

Figure 2. Homeostatic regulation has a weak influence on baboon sleep patterns. Neither the relative sleep

time on the previous night (the difference between an individual's total sleep time and its average total sleep time; A) nor the distance travelled on the preceding day (C) influenced sleep duration, although baboons did sleep less following days with more napping (B). Additionally, the likelihood of a baboon being asleep did not substantially decrease as the night progressed and the baboon payed off its sleep debt (D). In (D), time since the beginning of the sleep period is scaled from 0 (beginning) to 1 (end of the sleep period). Subplots depict conditional effects of each variable from models of the data, with raw data points overlaid.

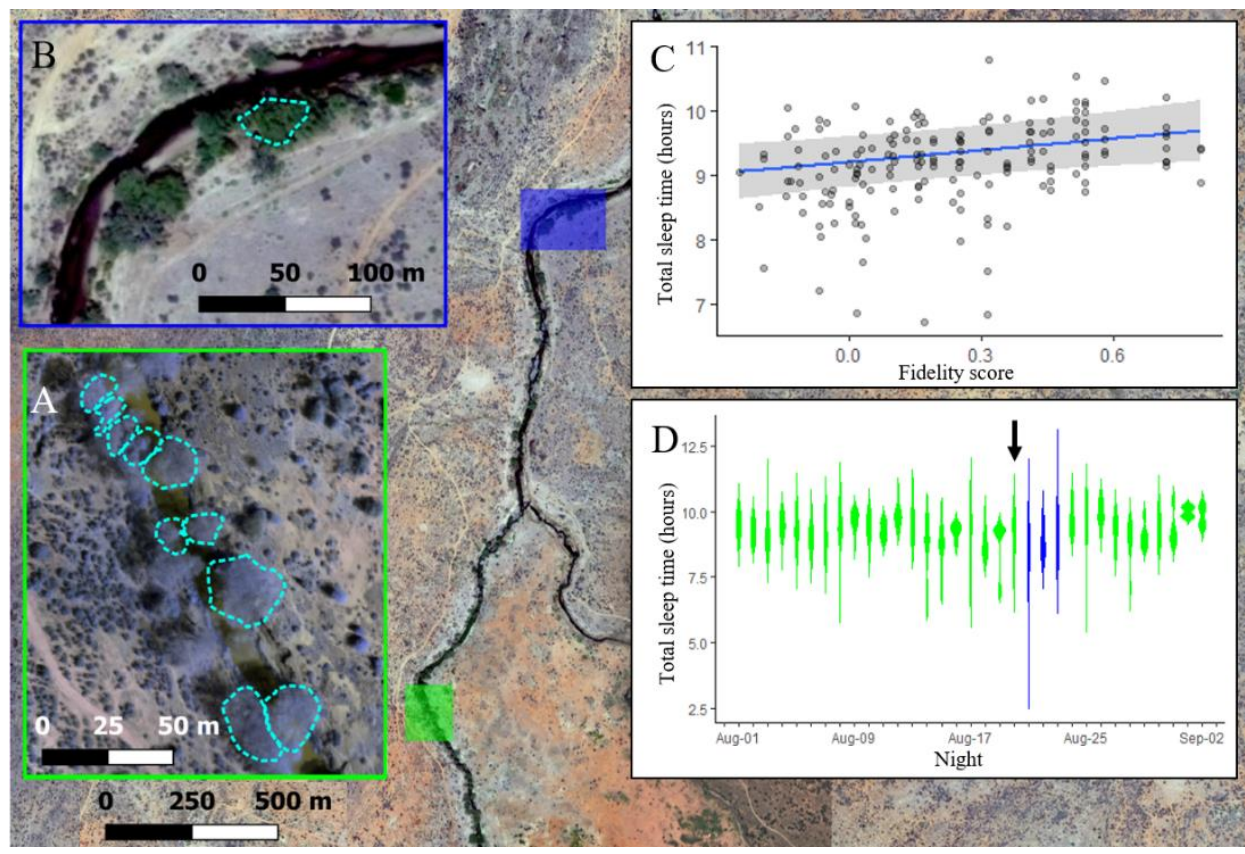

Figure 3. The location where baboons sleep has consequences on sleep duration. Group members spent the majority of the study (32/35 nights) sleeping in 10 yellow fever (A. xanthophloea) trees in a grove along the Ewaso Ng'iro river (A). Within this sleep site, baboons slept longer when sleeping in trees to which they showed high fidelity (C). At 20:30 on the $21^{\text {st }}$ night of the study, a leopard mounted an unsuccessful attack on the group in their sleep site. The following day, the baboons moved to a new sleep site $1.5 \mathrm{~km}$ away from their main sleep site (B). Baboons slept substantially less following this change in sleep site, but this effect did not persist beyond the first night in the new location (D). (C) depicts the conditional effects from models of the data, 

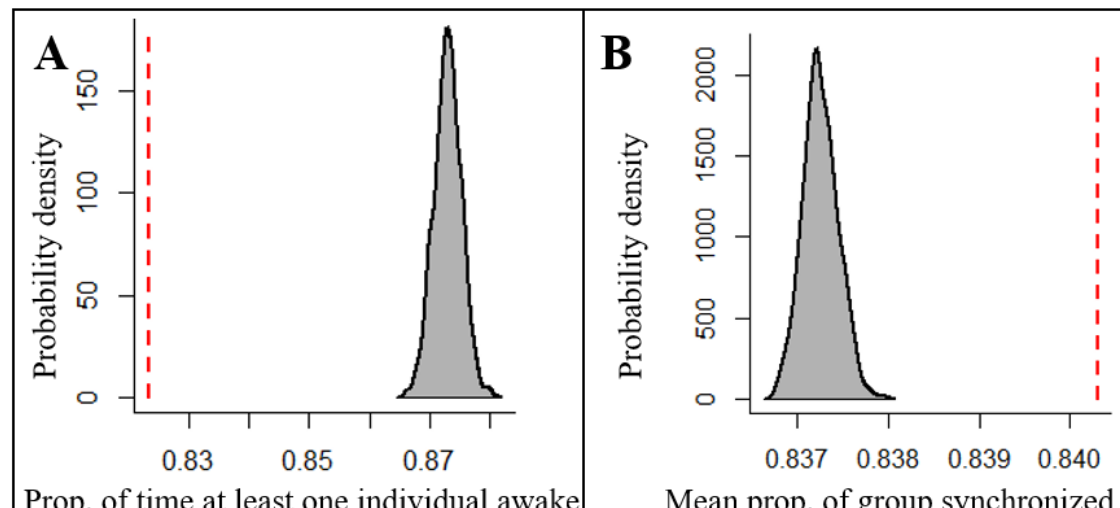

Prop. of time at least one individual awake Mean prop. of group synchronized

\section{Figure 4. Collective dynamics within the sleep site influence sleep patterns. Group-mates' periods of} nocturnal wakefulness were not staggered, but rather synchronized, as indicated by a significantly lower proportion of time with at least one individual awake (A, dotted red line; Fisher's exact test: $p<0.0001$ ) and a significantly greater proportion of the group exhibiting synchronized behaviors (B, dotted red line; Fisher's exact test: $\mathrm{p}<0.0001$ ) than expected based on 1000 time-shifted data sets (gray distribution). Synchronized sleep patterns likely result from individuals waking in response to the nighttime activity of nearby group-mates, as dyads show greater synchronization when dyad members sleep in the same tree compared to when they sleep in different trees (C). As a consequence of these local social perturbations, baboons sleep less when sleeping in trees with more group-mates (D). Subplots (C) and (D) depict the conditional effects from models of the data, with raw data points overlaid. 


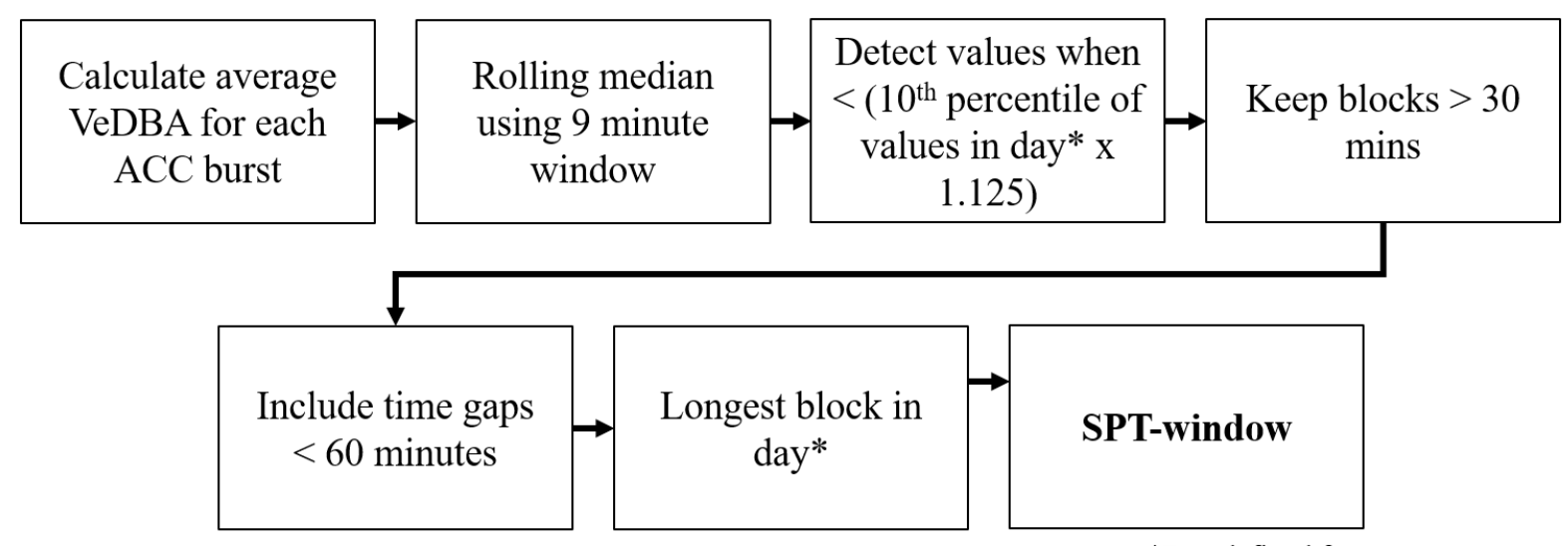

992

993

994

995

996

997

Figure 5. SPT-window detection algorithm adapted from van Hees et al., 2018.

*Day defined from noon to noon

\section{Supplemental Information}

\begin{tabular}{|c|c|c|c|c|}
\hline Collar \# & GPS start date & GPS end date & ACC start date & ACC end date \\
\hline $\mathbf{2 4 2 6}$ & $2012-08-01$ & $2012-08-30$ & $2012-08-01$ & $2012-08-31$ \\
\hline $\mathbf{2 4 2 7}$ & $2012-08-01$ & $2012-09-04$ & $2012-08-01$ & $2012-09-04$ \\
\hline $\mathbf{2 4 2 8}$ & $2012-08-01$ & $2012-08-15$ & $2012-08-01$ & $2012-08-15$ \\
\hline $\mathbf{2 4 3 0}$ & $2012-08-01$ & $2012-08-03$ & $2012-08-01$ & $2012-08-03$ \\
\hline $\mathbf{2 4 3 2}$ & $2012-08-01$ & $2012-08-05$ & $2012-08-01$ & $2012-08-05$ \\
\hline $\mathbf{2 4 3 3}$ & $2012-08-01$ & $2012-08-06$ & $2012-08-01$ & $2012-08-06$ \\
\hline $\mathbf{2 4 3 4}$ & $2012-08-01$ & $2012-08-02$ & $2012-08-01$ & $2012-08-02$ \\
\hline $\mathbf{2 4 3 6}$ & $2012-08-01$ & $2012-09-02$ & $2012-08-01$ & $2012-09-02$ \\
\hline $\mathbf{2 4 3 9}$ & $2012-08-01$ & $2012-09-04$ & $2012-08-01$ & $2012-09-04$ \\
\hline $\mathbf{2 4 4 1}$ & $2012-08-01$ & $2012-08-29$ & $2012-08-01$ & $2012-08-29$ \\
\hline $\mathbf{2 4 4 3}$ & $2012-08-01$ & $2012-09-02$ & $2012-08-01$ & $2012-09-02$ \\
\hline $\mathbf{2 4 4 6}$ & $2012-08-01$ & $2012-09-02$ & $2012-08-01$ & $2012-09-02$ \\
\hline $\mathbf{2 4 4 7}$ & $2012-08-01$ & $2012-08-31$ & $2012-08-01$ & $2012-08-31$ \\
\hline $\mathbf{2 4 4 8}$ & $2012-08-01$ & $2012-08-16$ & $2012-08-01$ & $2012-08-17$ \\
\hline $\mathbf{2 4 4 9}$ & $2012-08-01$ & $2012-08-31$ & $2012-08-01$ & $2012-08-31$ \\
\hline $\mathbf{2 4 5 0}$ & $2012-08-01$ & $2012-08-05$ & $2012-08-01$ & $2012-08-05$ \\
\hline $\mathbf{2 4 5 1}$ & $2012-08-01$ & $2012-09-02$ & $2012-08-01$ & $2012-09-02$ \\
\hline $\mathbf{2 4 5 2}$ & $2012-08-01$ & $2012-08-14$ & $2012-08-01$ & $2012-08-14$ \\
\hline $\mathbf{2 4 5 3}$ & $2012-08-01$ & $2012-08-03$ & $2012-08-01$ & $2012-08-03$ \\
\hline $\mathbf{2 4 5 4}$ & $2012-08-01$ & $2012-08-14$ & $2012-08-01$ & $2012-08-14$ \\
\hline $\mathbf{2 4 5 5}$ & $2012-08-01$ & $2012-08-08$ & $2012-08-01$ & $2012-08-08$ \\
\hline $\mathbf{2 4 5 6}$ & $2012-08-01$ & $2012-08-31$ & $2012-08-01$ & $2012-08-31$ \\
\hline $\mathbf{2 4 5 7}$ & $2012-08-01$ & $2012-09-04$ & $2012-08-01$ & $2012-09-04$ \\
\hline $\mathbf{2 4 5 8}$ & $2012-08-01$ & $2012-08-01$ & $2012-08-01$ & $2012-08-01$ \\
\hline $\mathbf{2 4 5 9}$ & $2012-08-01$ & $2012-08-01$ & $2012-08-01$ & $2012-08-01$ \\
\hline $\mathbf{2 4 6 0}$ & $2012-08-01$ & $2012-08-01$ & $2012-08-01$ & $2012-08-01$ \\
\hline $\mathbf{T a b} \mathbf{S 1}$ & Co & $\mathbf{2}-01$ & \\
\hline
\end{tabular}

Table S1. Collar metadata. Table depicts the data on which each collar began collecting data and ceased to collected data for both GPS and accelerometry (ACC). 


\begin{tabular}{|c|c|c|c|c|c|}
\hline & $\begin{array}{c}\text { Total sleep } \\
\text { time }\end{array}$ & $\begin{array}{l}\text { Sleep onset } \\
\text { time }\end{array}$ & $\begin{array}{l}\text { Waking } \\
\text { time }\end{array}$ & $\begin{array}{l}\text { Sleep period } \\
\text { duration }\end{array}$ & $\begin{array}{c}\text { Sleep } \\
\text { efficiency }\end{array}$ \\
\hline $\begin{array}{c}\text { Total sleep } \\
\text { time }\end{array}$ & 1 & -0.61 & 0.65 & 0.87 & 0.57 \\
\hline $\begin{array}{c}\text { Sleep onset } \\
\text { time }\end{array}$ & $\mathrm{x}$ & 1 & -0.04 & -0.72 & -0.05 \\
\hline Waking time & $\mathrm{X}$ & $\mathrm{X}$ & 1 & 0.72 & 0.10 \\
\hline $\begin{array}{l}\text { Sleep period } \\
\text { duration }\end{array}$ & $\mathrm{x}$ & $\mathrm{X}$ & $\mathrm{x}$ & 1 & 0.11 \\
\hline $\begin{array}{c}\text { Sleep } \\
\text { efficiency }\end{array}$ & $\mathrm{x}$ & $\mathrm{X}$ & $\mathrm{x}$ & $\mathrm{x}$ & 1 \\
\hline
\end{tabular}

Table S2. Pearson correlation coefficient between the metrics of sleep extracted from the

Histogram of total sleep time

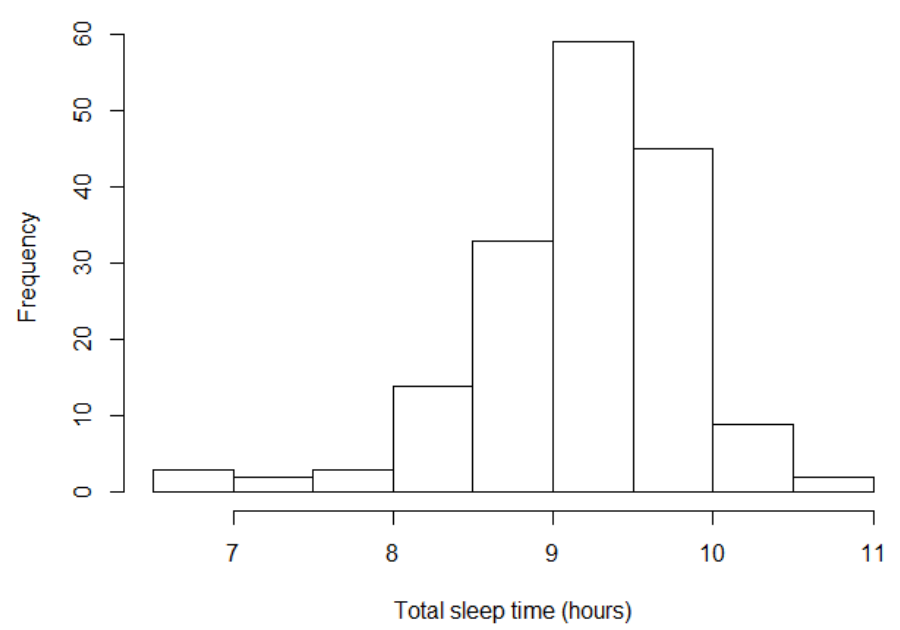

Figure S1. Histogram of total sleep time for the first 20 nights of the study (data prior to leopard attack

\begin{tabular}{|l|c|c|}
\hline & \multicolumn{2}{c|}{ Total sleep time (Standardized) } \\
\hline Predictors & Estimates & $C I(95 \%)$ \\
\hline Intercept & -0.03 & $-0.55-0.49$ \\
\hline Travel distance & -0.02 & $-0.18-0.15$ \\
\hline Time spent napping & -0.18 & $-0.36--0.01$ \\
\hline Previous night total sleep time & -0.00 & $-0.14-0.13$ \\
\hline Tree fidelity score & 0.20 & $0.05-0.36$ \\
\hline Relative number of baboons in tree & -0.51 & $-0.85--0.16$ \\
\hline Minimum ambient temperature & -0.03 & $-0.21-0.13$ \\
\hline Moon phase & 0.05 & $-0.12-0.22$ \\
\hline age: Juvenile & -0.32 & $-1.12-0.50$ \\
\hline age: Subadult & -0.32 & $-0.80-0.14$ \\
\hline sex: Male & 0.44 & $-0.04-0.92$ \\
\hline tree: tree2 & -0.26 & $-1.08-0.56$ \\
\hline tree: tree3 & -0.15 & $-1.01-0.69$ \\
\hline tree: tree4 & -0.81 & $-1.30--0.32$ \\
\hline
\end{tabular}




\begin{tabular}{|l|l|l|}
\hline tree: tree5 & -0.24 & $-0.74-0.27$ \\
\hline tree: tree6 & 0.02 & $-0.60-0.65$ \\
\hline tree: tree7 & 0.10 & $-0.62-0.84$ \\
\hline tree: tree8 & -0.26 & $-0.75-0.22$ \\
\hline tree: tree10 & -0.22 & $-0.95-0.51$ \\
\hline tree: tree11 & -0.22 & $-0.78-0.32$ \\
\hline Random Effects & 0.53 \\
\hline $\boldsymbol{\sigma}^{2}$ & 0.02 \\
\hline $\boldsymbol{\tau}_{\mathbf{0 0} \text { night }}$ & 0.08 \\
\hline $\boldsymbol{\tau}_{\mathbf{0 0}}$ tag & 0.16 \\
\hline ICC & 18 \\
\hline $\mathbf{N}_{\text {tag }}$ & 18 \\
\hline $\mathbf{N}_{\text {night }}$ & 170 \\
\hline Observations & $0.340 / 0.414$ \\
\hline $\mathbf{M a r g i n a l}^{\mathbf{2}} /$ Conditional $^{\mathbf{2}}$ & \\
\hline
\end{tabular}

Table S3. Model output table of model total sleep time (for the first 20 days) with all numerical variables standardized

Sex: Male

Age: Subadult

Age: Juvenile

Moon phase

Minimum ambient temperature

Relative number of baboons in tree

Tree fidelity score

Previous night total sleep time

Time spent napping

Travel distance

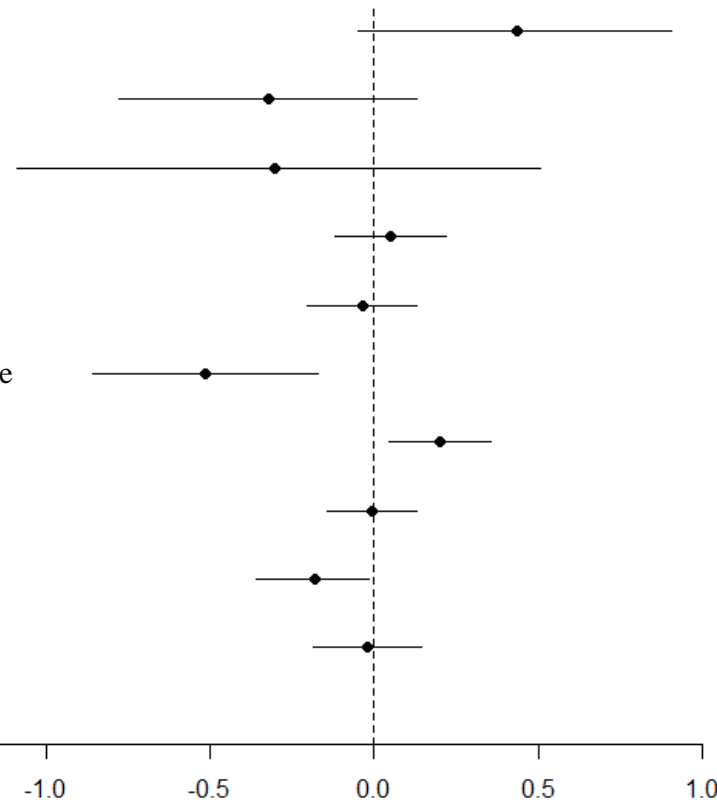

1010

Figure S2. Model output plot of model of total sleep time (for the first 20 days) with all numerical variables standardized. The categorical variable tree is not plotted

\begin{tabular}{|l|c|c|}
\hline & \multicolumn{2}{c|}{ Total sleep time (hours) } \\
\hline Predictors & Estimates & $C I(95 \%)$ \\
\hline Intercept & 18.65 & $-24.20-63.97$ \\
\hline Travel distance $\mathbf{( k m )}$ & -0.01 & $-0.12-0.09$ \\
\hline Time spent napping (mins) & -0.01 & $-0.01--0.00$ \\
\hline Previous night relative total sleep time (mins) & -0.00 & $-0.00-0.00$ \\
\hline Tree fidelity score & 0.60 & $0.15-1.03$ \\
\hline Relative number of baboons in tree & -1.55 & $-2.58--0.48$ \\
\hline
\end{tabular}




\begin{tabular}{|l|c|c|}
\hline Minimum ambient temperature (degree Celsius) & -0.03 & $-0.19-0.12$ \\
\hline Moon phase & 0.11 & $-0.25-0.48$ \\
\hline age: Juvenile & -0.26 & $-0.85-0.38$ \\
\hline age: Subadult & -0.25 & $-0.60-0.10$ \\
\hline sex: Male & 0.34 & $-0.03-0.72$ \\
\hline tree: tree2 & -0.20 & $-0.84-0.44$ \\
\hline tree: tree3 & -0.12 & $-0.79-0.55$ \\
\hline tree: tree4 & -0.64 & $-1.03--0.25$ \\
\hline tree: tree5 & -0.20 & $-0.59-0.19$ \\
\hline tree: tree6 & 0.02 & $-0.48-0.50$ \\
\hline tree: tree7 & 0.09 & $-0.46-0.64$ \\
\hline tree: tree8 & -0.22 & $-0.59-0.16$ \\
\hline tree: tree10 & -0.17 & $-0.74-0.38$ \\
\hline tree: tree11 & -0.18 & $-0.60-0.24$ \\
\hline Random Effects & & \\
\hline $\boldsymbol{\sigma}^{\mathbf{2}}$ & 0.31 \\
\hline $\boldsymbol{\tau}_{\mathbf{0 0}}$ night & 0.01 \\
\hline $\boldsymbol{\tau}_{\mathbf{0 0}}$ tag & 0.05 & \\
\hline ICC & 0.17 \\
\hline $\mathbf{N}_{\text {tag }}$ & 18 \\
\hline $\mathbf{N}_{\text {night }}$ & 18 \\
\hline Observations & 170 \\
\hline $\mathbf{M a r g i n a l}^{\mathbf{2}} /$ Conditional $\mathbf{R}^{\mathbf{2}}$ & $0.337 / 0.412$ \\
\hline
\end{tabular}

Table S4. Model output table of model total sleep time (for the first 20 days) with no standardization of variables

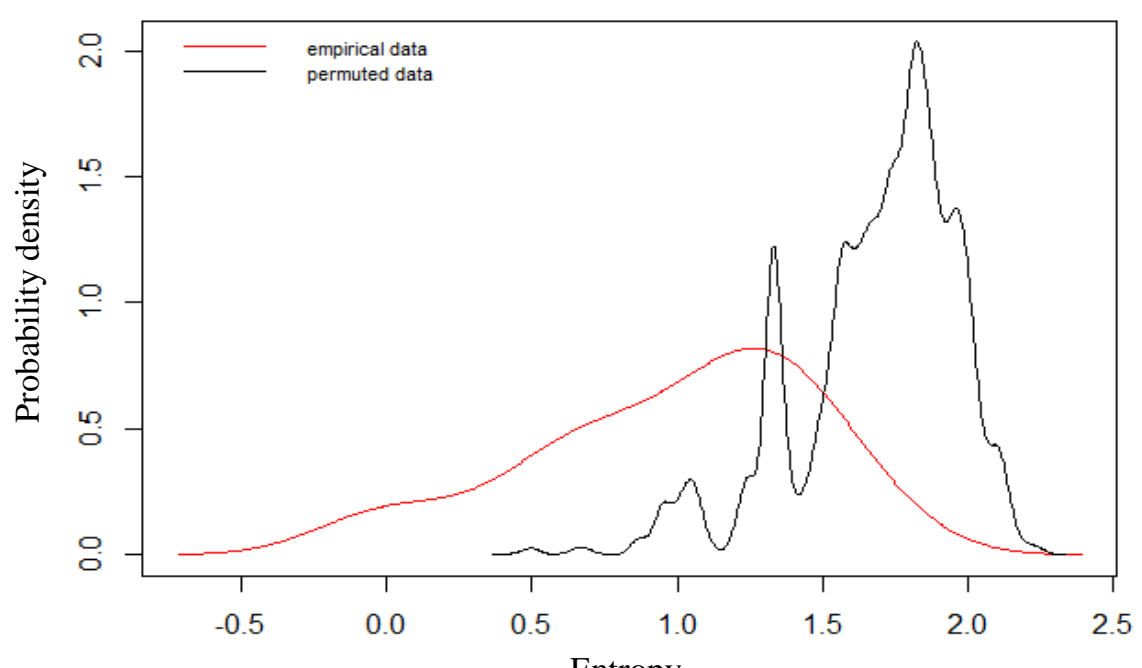

Figure S3. Comparison of the Shannon entropies of individuals' sleep tree occupancy within this sleep site to a null distribution produced by 1000 identity permutations. The analysis revealed lower entropy in tree occupancy than expected by random chance (one-tailed two-sample KolmogorovSmirnov test: $\left.\mathrm{p}<1.0 \times 10^{-9}\right)$, indicating that individuals exhibited high fidelity to particular trees. The red line represents the distribution of Shannon entropies of individuals' sleep tree occupancy calculated from the empirical data, and the black line represents the distribution of entropy of sleep tree occupancy derived from the permuted data set. 


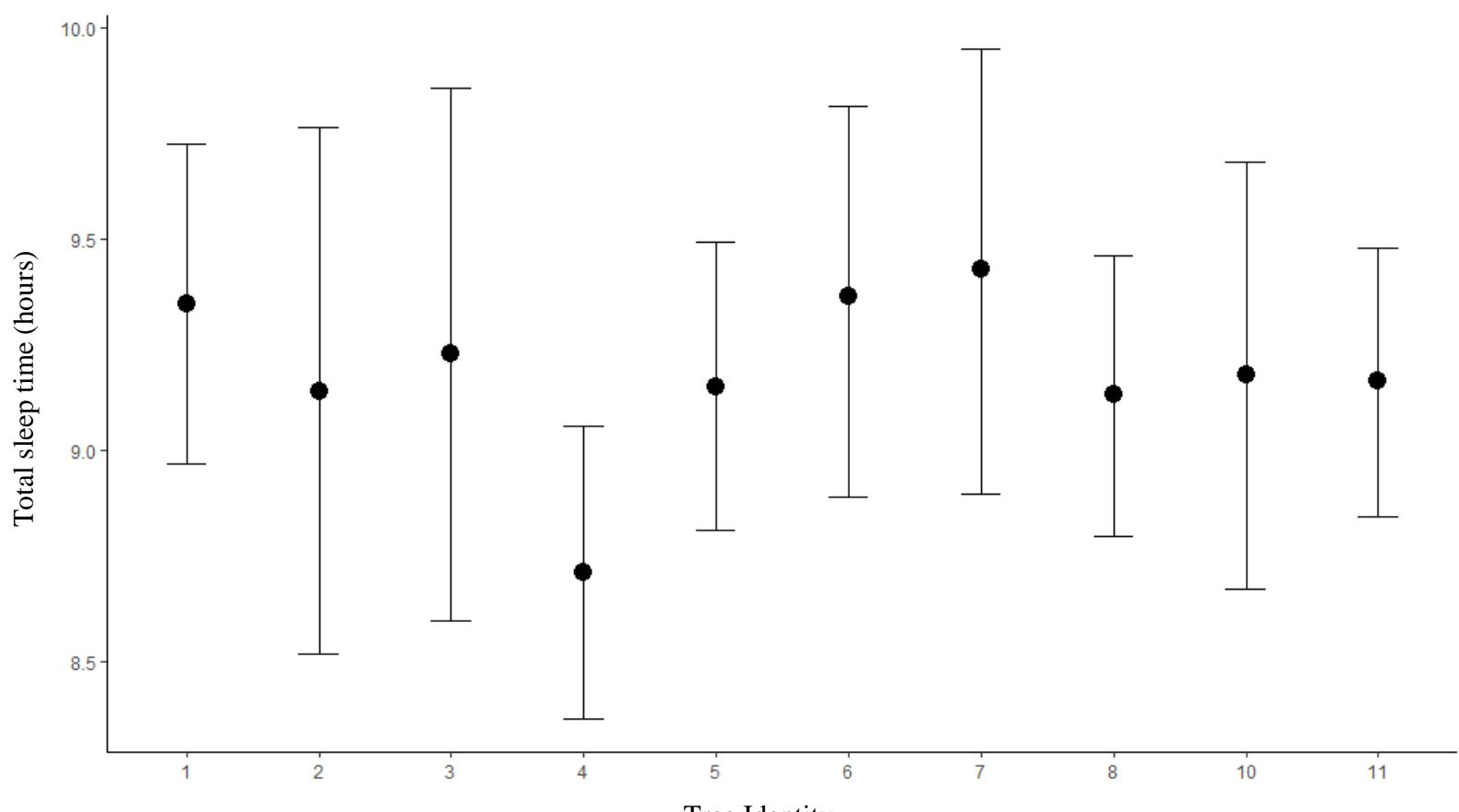

Tree Identity

Figure S4. The conditional effect of tree identity on total sleep time. The conditional effects plotted here are from the unstandardized Bayesian linear mixed model (LMM) of total sleep time (hours).

Total sleep time (Standarized)

\begin{tabular}{|l|c|c|}
\hline Predictors & Estimates & CI (95\%) \\
\hline Intercept & -0.04 & $-0.55-0.47$ \\
\hline Average VeDBA during day & -0.12 & $-0.33-0.12$ \\
\hline Time spent napping & -0.13 & $-0.30-0.05$ \\
\hline Previous night total sleep time & -0.00 & $-0.14-0.13$ \\
\hline Tree fidelity score & 0.20 & $0.04-0.36$ \\
\hline Relative number of baboons in tree & -0.55 & $-0.89--0.20$ \\
\hline Minimum ambient temperature & -0.04 & $-0.21-0.12$ \\
\hline Moon phase & 0.07 & $-0.09-0.23$ \\
\hline age: Juvenile & -0.21 & $-1.01-0.59$ \\
\hline age: Subadult & -0.26 & $-0.77-0.22$ \\
\hline sex: Male & 0.53 & $0.09-0.97$ \\
\hline tree: tree2 & -0.34 & $-1.18-0.49$ \\
\hline tree: tree3 & -0.47 & $-1.26-0.33$ \\
\hline tree: tree4 & -0.86 & $-1.37--0.35$ \\
\hline tree: tree5 & -0.28 & $-0.79-0.22$ \\
\hline tree: tree6 & 0.04 & $-0.60-0.68$ \\
\hline tree: tree7 & 0.08 & $-0.66-0.83$ \\
\hline tree: tree8 & -0.28 & $-0.77-0.23$ \\
\hline tree: tree10 & -0.13 & $-0.84-0.58$ \\
\hline tree: tree11 & -0.24 & $-0.79-0.31$ \\
\hline Random Effects & & \\
\hline $\boldsymbol{\sigma}^{2}$ & 0.55 & \\
\hline$\tau_{\mathbf{0 0}}$ night & 0.02 & \\
\hline$\tau_{00}$ tag & 0.07 & \\
\hline & & \\
\hline
\end{tabular}




\begin{tabular}{|l|l|}
\hline ICC & 0.13 \\
\hline $\mathbf{N}_{\text {tag }}$ & 18 \\
\hline $\mathbf{N}_{\text {night }}$ & 18 \\
\hline Observations & 178 \\
\hline Marginal $^{2}$ / Conditional $\mathbf{R}^{2}$ & $0.352 / 0.416$ \\
\hline
\end{tabular}

Table S5. Model output table of model total sleep time (for the first 20 days) with all numerical

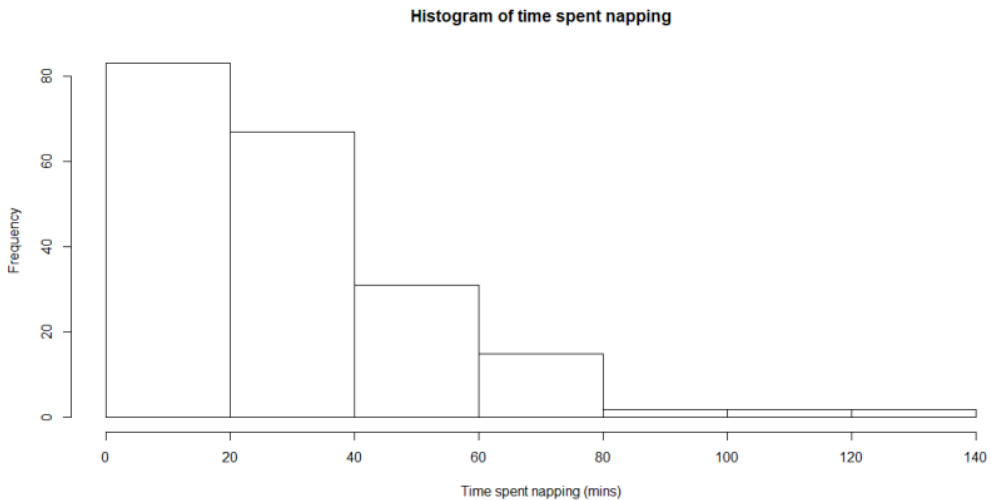

Figure S5. Histogram of time spent napping for the first 20 days of the study (data prior to leopard 1039 attack)

\begin{tabular}{|c|c|c|}
\hline & \multicolumn{2}{|c|}{ Time spent napping (standardized) } \\
\hline Predictors & Estimates & $C I(95 \%)$ \\
\hline Intercept & -0.10 & $-0.49-0.28$ \\
\hline Prior night total sleep time & 0.05 & $-0.08-0.18$ \\
\hline \multicolumn{3}{|l|}{ Random Effects } \\
\hline$\sigma^{2}$ & \multicolumn{2}{|l|}{0.41} \\
\hline$\tau_{00}$ night & \multicolumn{2}{|l|}{0.12} \\
\hline$\tau_{00 \text { tag }}$ & \multicolumn{2}{|l|}{0.51} \\
\hline ICC & \multicolumn{2}{|l|}{0.60} \\
\hline $\mathbf{N}_{\text {tag }}$ & \multicolumn{2}{|l|}{20} \\
\hline $\mathbf{N}_{\text {night }}$ & \multicolumn{2}{|l|}{19} \\
\hline Observations & \multicolumn{2}{|l|}{199} \\
\hline Marginal $\mathbf{R}^{2}$ / Conditional $\mathbf{R}^{2}$ & \multicolumn{2}{|l|}{$0.003 / 0.617$} \\
\hline
\end{tabular}

Table S6. Model output table of model of time spent napping during the day (for the first 20 days)

\begin{tabular}{|l|cc|}
\hline & \multicolumn{2}{c|}{ Time spent napping (minutes) } \\
\hline Predictors & Estimates & $C I(95 \%)$ \\
\hline Intercept & 21.22 & $-5.83-47.59$ \\
\hline Prior night total sleep time (hours) & 1.53 & $-1.23-4.42$ \\
\hline Random Effects & & \\
\hline $\boldsymbol{\sigma}^{\mathbf{2}}$ & 295.93 \\
\hline $\boldsymbol{\tau}_{\mathbf{0 0} \text { night }}$ & 25.86 \\
\hline $\boldsymbol{\tau}_{\mathbf{0 0} \text { tag }}$ & 99.07 \\
\hline $\mathbf{I C C}^{\mathbf{C}}$ & 0.30 \\
\hline $\mathbf{N}_{\text {tag }}$ & 20 \\
\hline $\mathbf{N}_{\text {night }}$ & 19 \\
\hline
\end{tabular}


1043

1044

1045

1046

\begin{tabular}{|l|l|}
\hline Observations & 199 \\
\hline Marginal $\mathbf{R}^{\mathbf{2}}$ / Conditional $\mathbf{R}^{\mathbf{2}}$ & 0.002 / 0.521 \\
\hline
\end{tabular}

Table S7. Model output table of model of time spent napping during the day (for the first 20 days) without standardization of the variables

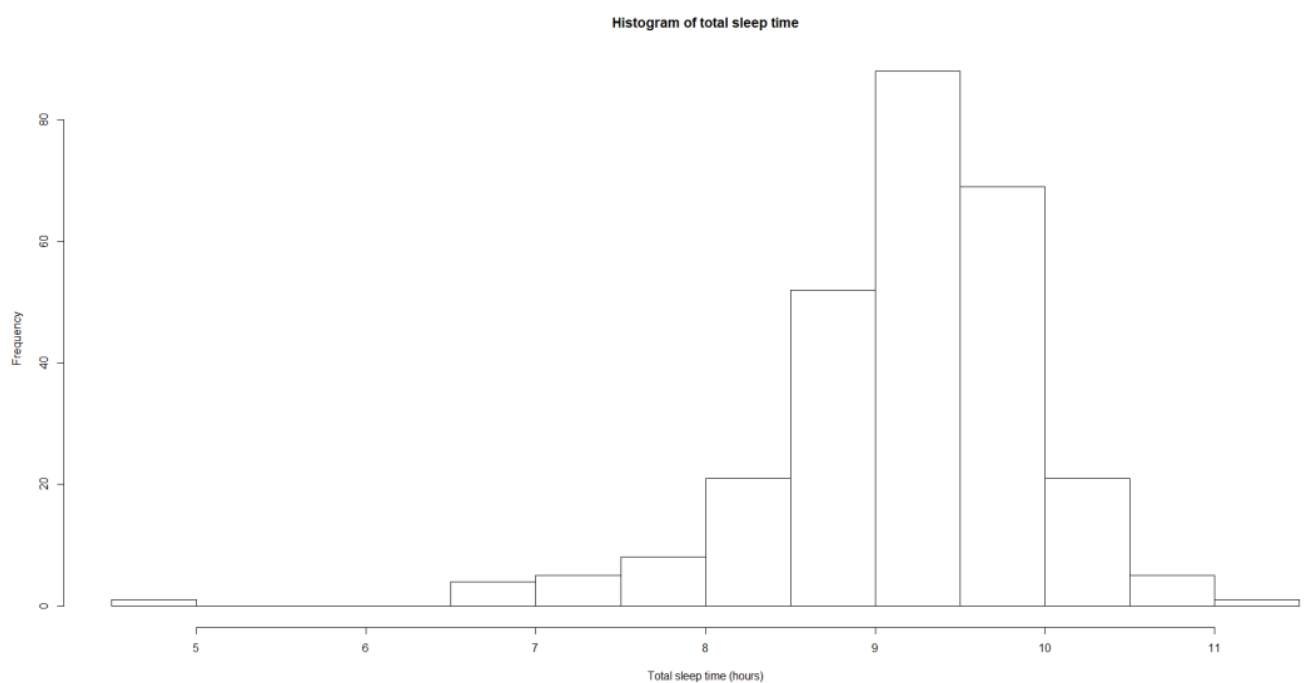

Figure S6. Histogram of total sleep time for the entire study duration

\begin{tabular}{|c|c|c|}
\hline & \multicolumn{2}{|c|}{ Total sleep time (standardized } \\
\hline Predictors & Estimates & $C I(95 \%)$ \\
\hline Intercept & -0.24 & $-0.57-0.11$ \\
\hline cond_night: night of leopard attack & -0.25 & $-0.87-0.39$ \\
\hline cond_night: first night in new sleep site & -1.55 & $-2.18--0.91$ \\
\hline cond_night: second night in new sleep site & -0.26 & $-0.98-0.44$ \\
\hline cond_night: third night in new sleep site & 0.21 & $-0.47-0.88$ \\
\hline cond_night: remainder of nights (in original sleep site) & 0.25 & $-0.07-0.56$ \\
\hline age: Juvenile & -0.27 & $-1.14-0.59$ \\
\hline age: Subadult & -0.36 & $-0.87-0.13$ \\
\hline sex: Male & 0.74 & $0.25-1.26$ \\
\hline Travel distance & -0.06 & $-0.18-0.07$ \\
\hline Time spent napping & -0.11 & $-0.28-0.05$ \\
\hline Previous night relative total sleep time & 0.15 & $0.04-0.27$ \\
\hline Minimum ambient temperature & -0.00 & $-0.14-0.14$ \\
\hline Moon phase & 0.02 & $-0.12-0.15$ \\
\hline \multicolumn{3}{|l|}{ Random Effects } \\
\hline$\sigma^{2}$ & \multicolumn{2}{|l|}{0.78} \\
\hline$\tau_{00 \text { tag }}$ & \multicolumn{2}{|l|}{0.14} \\
\hline ICC & \multicolumn{2}{|l|}{0.16} \\
\hline $\mathbf{N}_{\text {tag }}$ & \multicolumn{2}{|l|}{20} \\
\hline Observations & \multicolumn{2}{|l|}{275} \\
\hline Marginal $\mathbf{R}^{2}$ / Conditional $\mathbf{R}^{2}$ & \multicolumn{2}{|l|}{$0.251 / 0.314$} \\
\hline
\end{tabular}

Table S8. Model output table of model of total sleep time using data from entire study duration (including after the leopard attack) with all variables standardized 


\begin{tabular}{|c|c|c|}
\hline Predictors & Estimates & $C I(95 \%)$ \\
\hline Intercept & 8.43 & $-43.81-59.58$ \\
\hline cond_night: night of leopard attack & -0.21 & $-0.90-0.48$ \\
\hline cond_night: first night in new sleep site & -1.19 & $-1.88--0.49$ \\
\hline cond_night: second night in new sleep site & -0.23 & $-0.98-0.48$ \\
\hline cond_night: third night in new sleep site & 0.15 & $-0.58-0.86$ \\
\hline cond_night: remainder of nights (in original sleep site) & 0.15 & $-0.19-0.48$ \\
\hline age: Juvenile & -0.28 & $-0.96-0.39$ \\
\hline age: Subadult & -0.31 & $-0.71-0.07$ \\
\hline sex: Male & 0.58 & $0.19-0.99$ \\
\hline Travel distance & -0.04 & $-0.13-0.05$ \\
\hline Time spent napping & -0.00 & $-0.01-0.00$ \\
\hline Previous night relative total sleep time & 0.00 & $0.00-0.00$ \\
\hline Minimum ambient temperature & 0.00 & $-0.17-0.19$ \\
\hline Moon phase & 0.07 & $-0.33-0.47$ \\
\hline \multicolumn{3}{|l|}{ Random Effects } \\
\hline$\sigma^{2}$ & \multicolumn{2}{|l|}{0.43} \\
\hline$\tau_{00}$ night & \multicolumn{2}{|l|}{0.05} \\
\hline$\tau_{00} \operatorname{tag}$ & \multicolumn{2}{|l|}{0.09} \\
\hline ICC & \multicolumn{2}{|l|}{0.25} \\
\hline $\mathbf{N}_{\text {tag }}$ & \multicolumn{2}{|l|}{20} \\
\hline $\mathbf{N}_{\text {night }}$ & \multicolumn{2}{|l|}{32} \\
\hline Observations & \multicolumn{2}{|l|}{275} \\
\hline Marginal $\mathbf{R}^{2}$ / Conditional $\mathbf{R}^{2}$ & \multicolumn{2}{|c|}{$0.257 / 0.365$} \\
\hline
\end{tabular}

Table S9. Model output table of model of total sleep time using data from entire study duration (including after the leopard attack) without standardization of variables
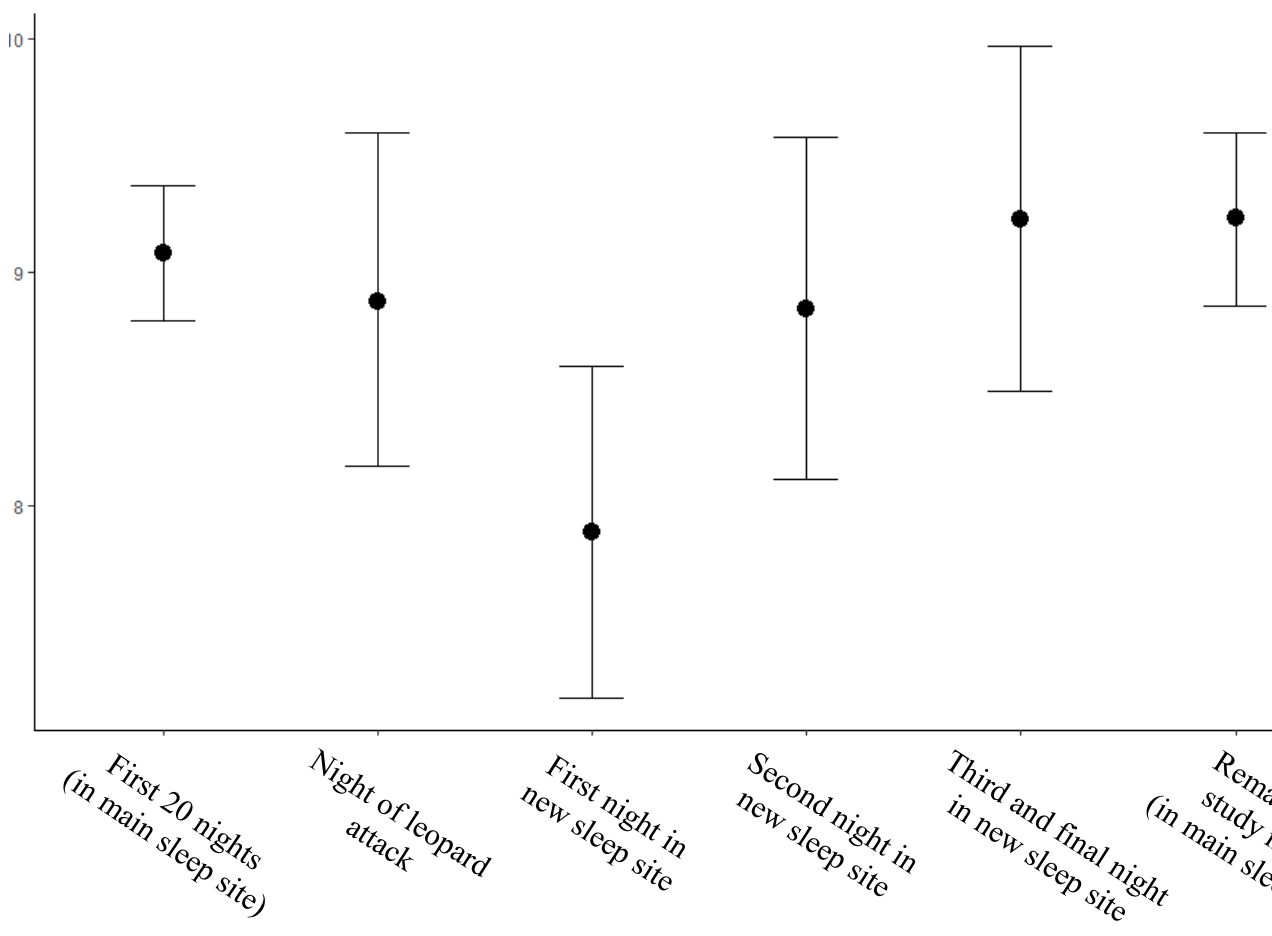

Figure S7. The conditional effect of night condition on total sleep time. The conditional effects presented here are from the unstandardized model of total sleep time. 


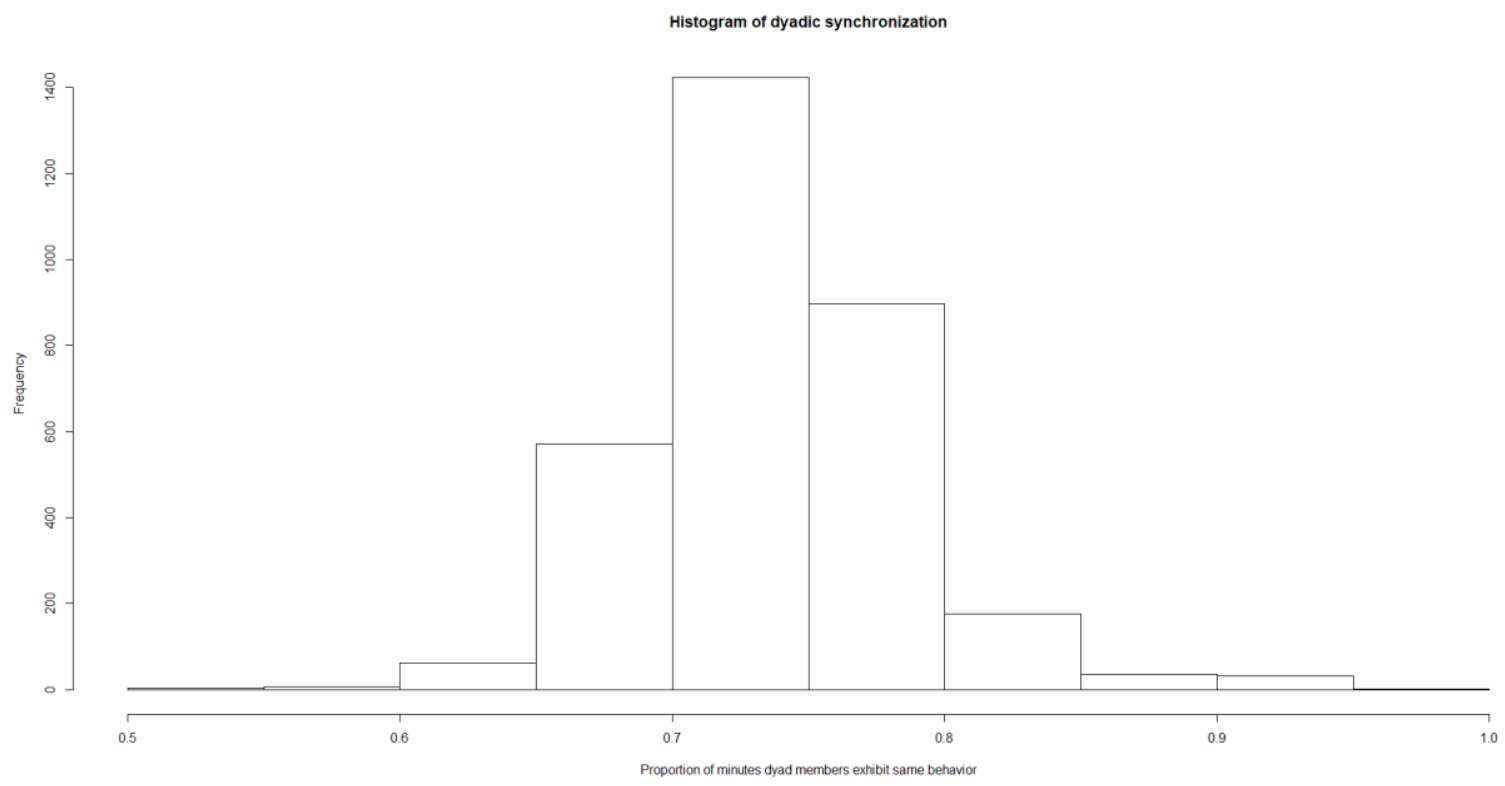

Figure S8. Histogram of the dyadic synchronization scores, which indicates the proportions of minutes between 21:00 and 05:00 during which both members of a dyad exhibited the same behavior (either sleep or wakefulness) on a given night.

1068

\begin{tabular}{|c|c|c|}
\hline 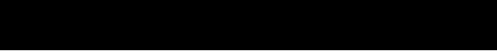 & \multicolumn{2}{|c|}{ Proportion of minutes synchronized (Standardized) } \\
\hline Predictors & Estimates & CI $(95 \%)$ \\
\hline Intercept & -0.21 & $-0.45-0.03$ \\
\hline Occupying same tree & 0.56 & $0.47-0.64$ \\
\hline \multicolumn{3}{|l|}{ Random Effects } \\
\hline$\sigma^{2}$ & 0.60 & \\
\hline$\tau_{00}$ dy_name & 0.14 & \\
\hline$\tau_{00}$ night & 0.19 & \\
\hline$\tau_{00} \operatorname{tag} 1$ & 0.08 & \\
\hline$\tau_{00 \text { tag2 }}$ & 0.09 & \\
\hline ICC & 0.45 & \\
\hline $\mathbf{N}_{\text {night }}$ & 34 & \\
\hline $\mathbf{N}_{\text {tag1 }}$ & 22 & \\
\hline $\mathbf{N}_{\text {tag2 }}$ & 22 & \\
\hline $\mathbf{N}_{\text {dy_name }}$ & 250 & \\
\hline Observations & 2997 & \\
\hline Marginal $\mathbf{R}^{2}$ / Conditional $\mathbf{R}^{2}$ & $0.050 / 0.404$ & \\
\hline
\end{tabular}

1069 Table S10. Model output table of model of synchronization (i.e. the proportion of minutes during a 1070 night that both dyad members exhibit the same behavior, either sleep or wakefulness) with response variable standardized of the response variable

\begin{tabular}{|l|c|c|}
\hline \multicolumn{2}{|c|}{ Proportion of minutes synchronized } \\
\hline Predictors & Estimates & CI (95\%) \\
\hline Intercept & 0.73 & $0.71-0.74$ \\
\hline Occupying same tree & 0.03 & $0.02-0.03$ \\
\hline Random Effects & \multicolumn{2}{|}{} \\
\hline $\boldsymbol{\sigma}^{\mathbf{2}}$ & 0.00 \\
\hline $\boldsymbol{\tau}_{\mathbf{0} \text { dy_name }}$ & 0.00 \\
\hline $\boldsymbol{\tau}_{\mathbf{0 0} \text { night }}$ & 0.00 & \\
\hline
\end{tabular}


bioRxiv preprint doi: https://doi.org/10.1101/2021.09.26.461870; this version posted September 27, 2021. The copyright holder for this preprint (which was not certified by peer review) is the author/funder, who has granted bioRxiv a license to display the preprint in perpetuity. It is made available under aCC-BY 4.0 International license.

1073

\begin{tabular}{|l|l|}
\hline $\boldsymbol{\tau}_{\mathbf{0 0} \text { tag1 }}$ & 0.00 \\
\hline $\boldsymbol{\tau}_{\mathbf{0 0} \text { tag2 }}$ & 0.00 \\
\hline $\mathbf{I C C}$ & 0.45 \\
\hline $\mathbf{N}_{\text {night }}$ & 34 \\
\hline $\mathbf{N}_{\text {tag1 }}$ & 22 \\
\hline $\mathbf{N}_{\text {tag2 }}$ & 22 \\
\hline $\mathbf{N}_{\text {dy_name }}$ & 250 \\
\hline Observations & 2997 \\
\hline Marginal $\mathbf{R}^{2} /$ Conditional $\mathbf{R}^{2}$ & $0.050 / 0.404$ \\
\hline
\end{tabular}

Table S11. Model output table of model of synchronization (i.e. the proportion of minutes during a night that both dyad members exhibit the same behavior, either sleep or wakefulness) without standardization of the response variable

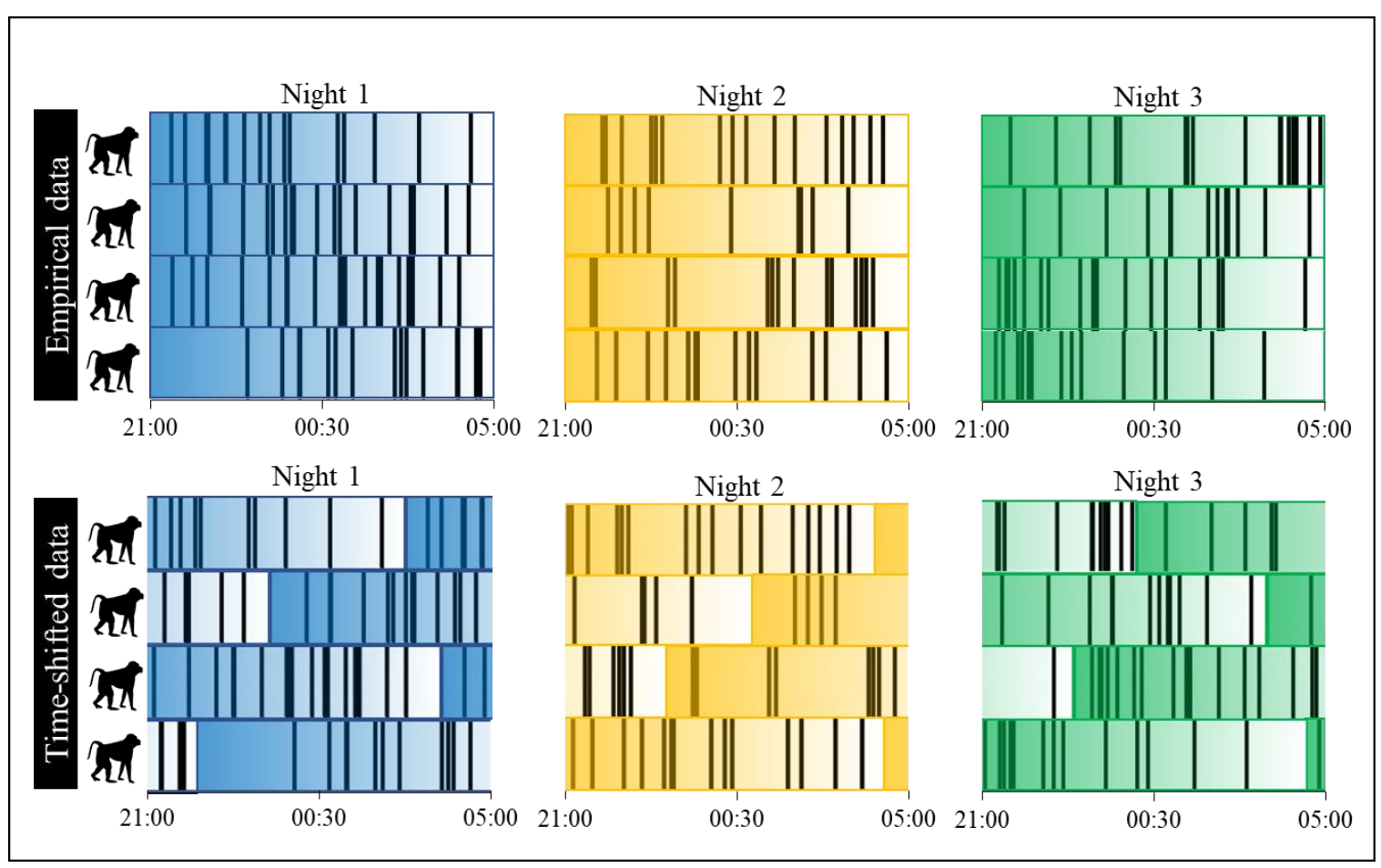

Figure S9. A toy example of the procedure we used to test for sentinel behavior and synchronization of nighttime behavior. Each row represents a baboon's time-series of sleep and wake activity during the night, with black vertical lines indicating periods of nocturnal waking behavior. Colors correspond to different nights, and the transparency of the color indicates the timing of night, with reference to the empirical, unshifted data. The time shifting procedure was repeated 1000 times to generate a null distribution for the proportion of minutes in which at least one individual is awake during the night and the mean proportion of the group exhibiting synchronized behavior. 


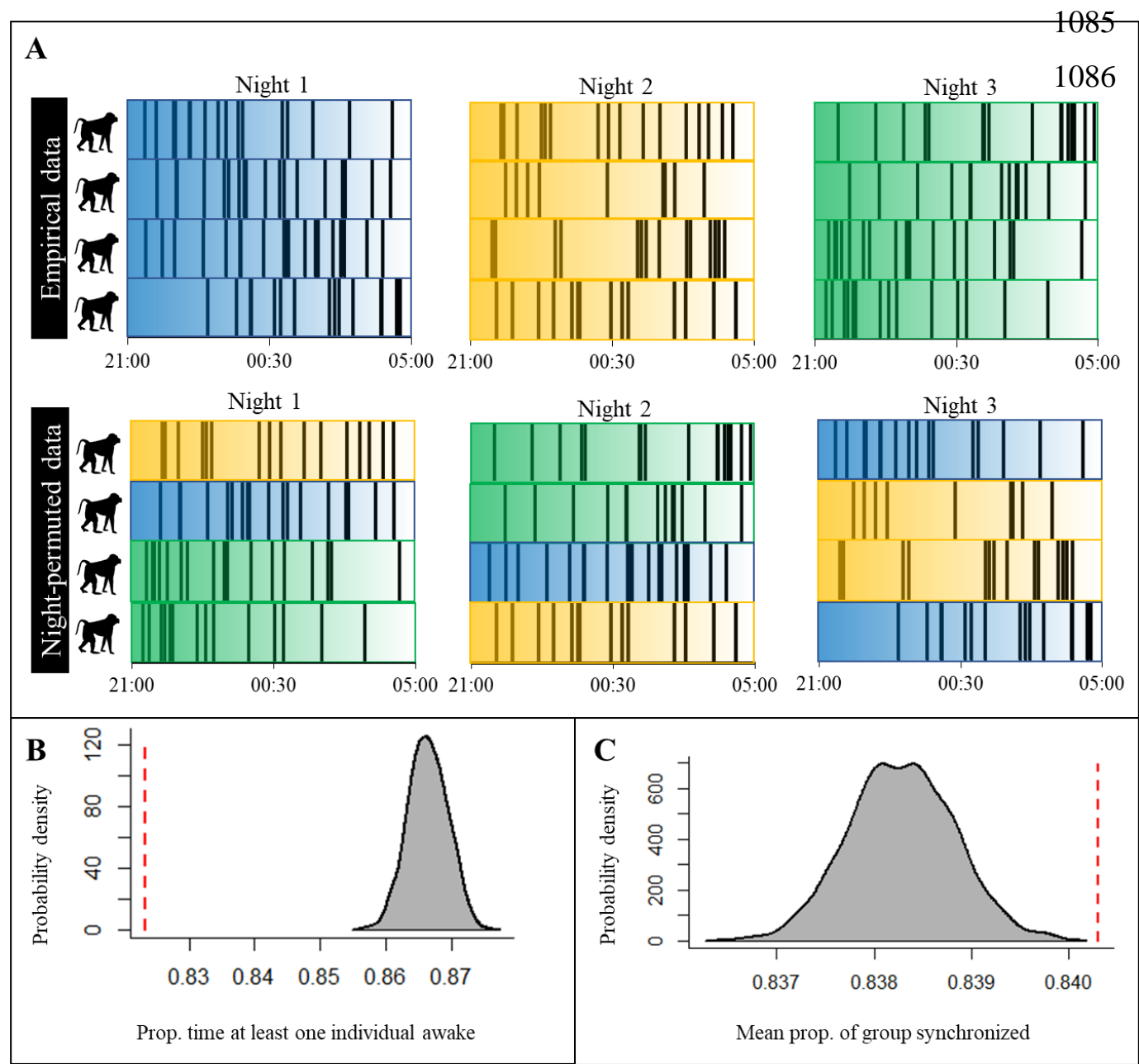

Figure S10. A) A toy example of the procedure alternative to the one presented in the main text (and represented in Figure S9) that we used to confirm findings concerning sentinel behavior and synchronization of nighttime behavior that we derived from the procedure presented in the main text. Each row represents a baboon's time-series of sleep and wake activity during the night, with black vertical lines indicating periods of nocturnal waking behavior. Colors correspond to different nights, with reference to the empirical, unpermuted data, and the transparency of the color indicates the timing of night. The night permutation procedure was repeated 1000 times to generate a null distribution for the proportion of minutes in which at least one individual is awake during the night and the mean proportion of the group exhibiting synchronized behavior. B) Comparison of the empirical proportion of minutes in which at least one individual is awake (red dotted line) to its null distribution (grey density plot; $p<0.0001$ ). C) Comparison of the empirical mean of the proportion of the group exhibiting synchronized behavior (red dotted line) to its null distribution (grey density plot; $\mathrm{p}<0.0001$ ). This method of permutation controls for the possibility that baboons are synchronized in their behavior simply as a result of species-typical nocturnal waking patterns that are consistent across baboons and across nights.

\section{Sleep validation study}

To evaluate whether the accelerometer-based sleep classification technique was accurately monitoring sleep in baboons, we returned to Mpala Research Centre in July 2019 to perform a validation study in which we compared the results of the accelerometer-based sleep classification to direct observations of awake and sleeping baboons. Using the procedures described in StrandburgPeshkin et al., 2015, we trapped and anesthetized 27 members of a group of habituated olive baboons, fitting each with a GPS and accelerometry collar. Eleven of the 27 collars deployed recorded continuous tri-axial accelerations at $12 \mathrm{~Hz} /$ axis from 06:30 to 18:00 and 0.71-second bursts of 
accelerations at $56.2 \mathrm{~Hz} /$ axis at the beginning of every minute from 18:00 to 06:30. Accelerometry data was collected by each of these 11 collars for up to 31 days. The remaining 16 collars did not collect accelerometry data from 06:30 to 18:00, and thus we excluded data from these collars from the validation study.

We down-sampled and interpolated the accelerometry data such that it matched the sampling frequency and schedule of the data collected in 2012 (i.e. the data analyzed for this manuscript). We then applied the sleep classification algorithm described in the Materials and Methods to this validation dataset.

To validate the sleep classification algorithm, we performed direct behavioral observations of the baboons at their primary sleep site. We recorded the behavior of the study baboons starting when they approached their sleep site using a FLIR T1020 high-resolution infrared camera (FLIR Systems Inc., Wilsonville, OR, USA). Recordings continued into the night for as long as the camera battery allowed (average recording duration (range of recording durations): 7.4 hours (1.7 - 14.9 hours)), and we collected thermal imaging data on 21 nights. We identified individuals in the thermal imagery both in real-time, via observer narration of the recorded imagery, and post-recording, by matching movements of individuals in the thermal imagery to the GPS tracks of collared individuals.

Following initial data collection, we used the commercial software Loopy (Loopbio GmbH, Austria) to score the behavior of identified individuals in the thermal imagery. Individuals' behavior was scored as "wakefulness", "resting wakefulness", or "sleep". Wakefulness refers to any behavior involving active movement (i.e. walking, running) or engaged activity (i.e. allogrooming), whereas resting wakefulness refers to behaviors that are dormant (i.e. sitting), but not in the typical sleeping posture of a baboon (sitting or lying with neck relaxed and head hung). Sustained dormant behavior in the typical sleep posture was considered sleep. Video scoring resulted in a total of 8.0 hours of behavioral observation across a total of 16 individual baboons.

Synchronizing the thermal imagery data with the accelerometry data produced a validation dataset of 294 minute-epochs across six baboons that were both classified as either sleep or wakeful behavior from accelerometry, and scored as wakefulness, resting wakefulness, or sleep from direct observation. With both wakefulness and resting wakefulness representing wakeful behavior, the accelerometer-based sleep classification exhibited an accuracy of $79.9 \%$ (Table S12). Consistent with previous validation studies of the use of accelerometry in measuring sleep (Ancoli-Israel et al., 2003; de Souza et al., 2003), we found that accelerometer-based sleep classification has difficulty distinguishing resting wakefulness from sleep, and we consider this limitation in our interpretation of the results.

\begin{tabular}{|c|c|c|c|c|}
\hline & & & havioral scori & \\
\hline & & & & Asleep \\
\hline & & Wakefulness & $\begin{array}{c}\text { Resting } \\
\text { wakefulness }\end{array}$ & Sleep \\
\hline Accelerometer- & Awake & 30 & 85 & 19 \\
\hline classification & Asleep & 0 & 40 & 120 \\
\hline
\end{tabular}
number of minute-epochs classified according to the accelerometer-based technique and direct

1149 behavioral observation. 


\section{Supplemental Information}

\begin{tabular}{|c|c|c|c|c|}
\hline Collar \# & GPS start date & GPS end date & ACC start date & ACC end date \\
\hline 2426 & 2012-08-01 & $2012-08-30$ & 2012-08-01 & 2012-08-31 \\
\hline 2427 & 2012-08-01 & 2012-09-04 & 2012-08-01 & 2012-09-04 \\
\hline 2428 & 2012-08-01 & 2012-08-15 & 2012-08-01 & $2012-08-15$ \\
\hline 2430 & 2012-08-01 & 2012-08-03 & 2012-08-01 & 2012-08-03 \\
\hline 2432 & 2012-08-01 & 2012-08-05 & 2012-08-01 & 2012-08-05 \\
\hline 2433 & 2012-08-01 & 2012-08-06 & 2012-08-01 & 2012-08-06 \\
\hline 2434 & 2012-08-01 & 2012-08-02 & 2012-08-01 & 2012-08-02 \\
\hline 2436 & 2012-08-01 & 2012-09-02 & 2012-08-01 & 2012-09-02 \\
\hline 2439 & 2012-08-01 & 2012-09-04 & 2012-08-01 & 2012-09-04 \\
\hline 2441 & 2012-08-01 & 2012-08-29 & 2012-08-01 & 2012-08-29 \\
\hline 2443 & 2012-08-01 & 2012-09-02 & 2012-08-01 & 2012-09-02 \\
\hline 2446 & 2012-08-01 & 2012-09-02 & 2012-08-01 & 2012-09-02 \\
\hline 2447 & 2012-08-01 & 2012-08-31 & 2012-08-01 & 2012-08-31 \\
\hline 2448 & 2012-08-01 & 2012-08-16 & 2012-08-01 & $2012-08-17$ \\
\hline 2449 & 2012-08-01 & 2012-08-31 & 2012-08-01 & 2012-08-31 \\
\hline 2450 & 2012-08-01 & 2012-08-05 & 2012-08-01 & 2012-08-05 \\
\hline 2451 & 2012-08-01 & 2012-09-02 & 2012-08-01 & 2012-09-02 \\
\hline 2452 & 2012-08-01 & 2012-08-14 & 2012-08-01 & 2012-08-14 \\
\hline 2453 & 2012-08-01 & 2012-08-03 & 2012-08-01 & 2012-08-03 \\
\hline 2454 & 2012-08-01 & 2012-08-14 & 2012-08-01 & 2012-08-14 \\
\hline 2455 & 2012-08-01 & 2012-08-08 & 2012-08-01 & 2012-08-08 \\
\hline 2456 & 2012-08-01 & 2012-08-31 & 2012-08-01 & 2012-08-31 \\
\hline 2457 & 2012-08-01 & 2012-09-04 & 2012-08-01 & 2012-09-04 \\
\hline 2458 & 2012-08-01 & 2012-08-01 & 2012-08-01 & 2012-08-01 \\
\hline 2459 & 2012-08-01 & 2012-08-01 & 2012-08-01 & 2012-08-01 \\
\hline 2460 & 2012-08-01 & 2012-08-01 & 2012-08-01 & 2012-08-01 \\
\hline
\end{tabular}

Table S1. Collar metadata. Table depicts the data on which each collar began collecting data and ceased to collected data for both GPS and accelerometry (ACC).

\begin{tabular}{|c|c|c|c|c|c|}
\hline & $\begin{array}{l}\text { Total sleep } \\
\text { time }\end{array}$ & $\begin{array}{l}\text { Sleep onset } \\
\text { time }\end{array}$ & $\begin{array}{c}\text { Waking } \\
\text { time }\end{array}$ & $\begin{array}{l}\text { Sleep period } \\
\text { duration }\end{array}$ & $\begin{array}{c}\text { Sleep } \\
\text { efficiency }\end{array}$ \\
\hline $\begin{array}{l}\text { Total sleep } \\
\text { time }\end{array}$ & 1 & -0.61 & 0.65 & 0.87 & 0.57 \\
\hline $\begin{array}{c}\text { Sleep onset } \\
\text { time }\end{array}$ & $\mathrm{x}$ & 1 & -0.04 & -0.72 & -0.05 \\
\hline Waking time & $\mathrm{x}$ & $\mathrm{x}$ & 1 & 0.72 & 0.10 \\
\hline $\begin{array}{c}\text { Sleep period } \\
\text { duration }\end{array}$ & $\mathrm{x}$ & $\mathrm{x}$ & $\mathrm{x}$ & 1 & 0.11 \\
\hline $\begin{array}{c}\text { Sleep } \\
\text { efficiency }\end{array}$ & $\mathrm{x}$ & $\mathrm{x}$ & $\mathrm{x}$ & $\mathrm{x}$ & 1 \\
\hline
\end{tabular}

Table S2. Pearson correlation coefficient between the metrics of sleep extracted from the 


\section{Histogram of total sleep time}

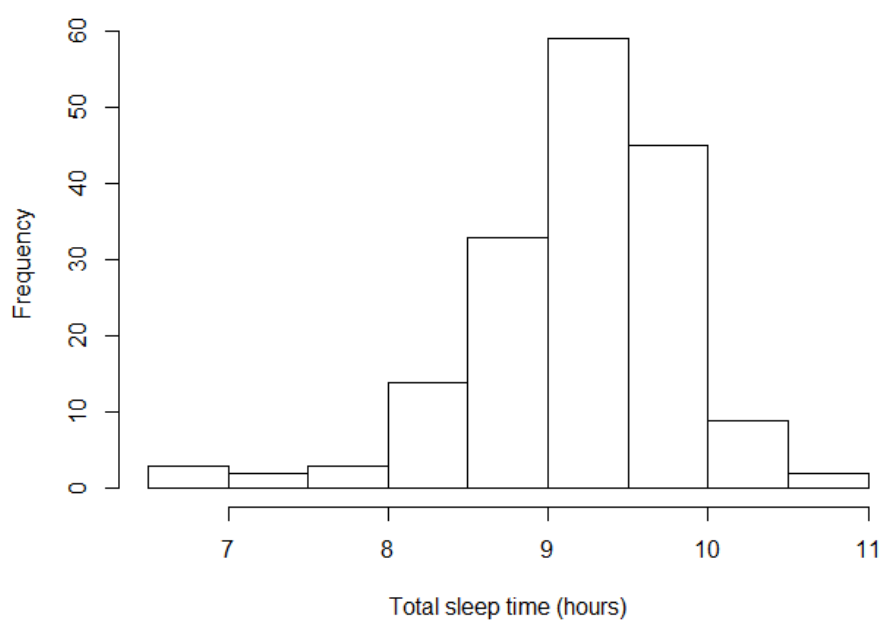

Figure S1. Histogram of total sleep time for the first 20 nights of the study (data prior to leopard attack

\begin{tabular}{|c|c|c|}
\hline & \multicolumn{2}{|c|}{ Total sleep time (Standardized) } \\
\hline Predictors & Estimates & $C I(95 \%)$ \\
\hline Intercept & -0.03 & $-0.55-0.49$ \\
\hline Travel distance & -0.02 & $-0.18-0.15$ \\
\hline Time spent napping & -0.18 & $-0.36--0.01$ \\
\hline Previous night total sleep time & -0.00 & $-0.14-0.13$ \\
\hline Tree fidelity score & 0.20 & $0.05-0.36$ \\
\hline Relative number of baboons in tree & -0.51 & $-0.85--0.16$ \\
\hline Minimum ambient temperature & -0.03 & $-0.21-0.13$ \\
\hline Moon phase & 0.05 & $-0.12-0.22$ \\
\hline age: Juvenile & -0.32 & $-1.12-0.50$ \\
\hline age: Subadult & -0.32 & $-0.80-0.14$ \\
\hline sex: Male & 0.44 & $-0.04-0.92$ \\
\hline tree: tree 2 & -0.26 & $-1.08-0.56$ \\
\hline tree: tree 3 & -0.15 & $-1.01-0.69$ \\
\hline tree: tree 4 & -0.81 & $-1.30--0.32$ \\
\hline tree: tree5 & -0.24 & $-0.74-0.27$ \\
\hline tree: tree6 & 0.02 & $-0.60-0.65$ \\
\hline tree: tree7 & 0.10 & $-0.62-0.84$ \\
\hline tree: tree8 & -0.26 & $-0.75-0.22$ \\
\hline tree: tree10 & -0.22 & $-0.95-0.51$ \\
\hline tree: tree11 & -0.22 & $-0.78-0.32$ \\
\hline \multicolumn{3}{|l|}{ Random Effects } \\
\hline$\sigma^{2}$ & \multicolumn{2}{|l|}{0.53} \\
\hline$\tau_{00}$ night & \multicolumn{2}{|l|}{0.02} \\
\hline$\tau_{00 \text { tag }}$ & \multicolumn{2}{|l|}{0.08} \\
\hline ICC & \multicolumn{2}{|l|}{0.16} \\
\hline $\mathbf{N}_{\text {tag }}$ & \multicolumn{2}{|l|}{18} \\
\hline $\mathbf{N}_{\text {night }}$ & \multicolumn{2}{|l|}{18} \\
\hline Observations & \multicolumn{2}{|l|}{170} \\
\hline Marginal $\mathbf{R}^{2}$ / Conditional $\mathbf{R}^{2}$ & \multicolumn{2}{|l|}{$0.340 / 0.414$} \\
\hline
\end{tabular}


Table S3. Model output table of model total sleep time (for the first 20 days) with all numerical

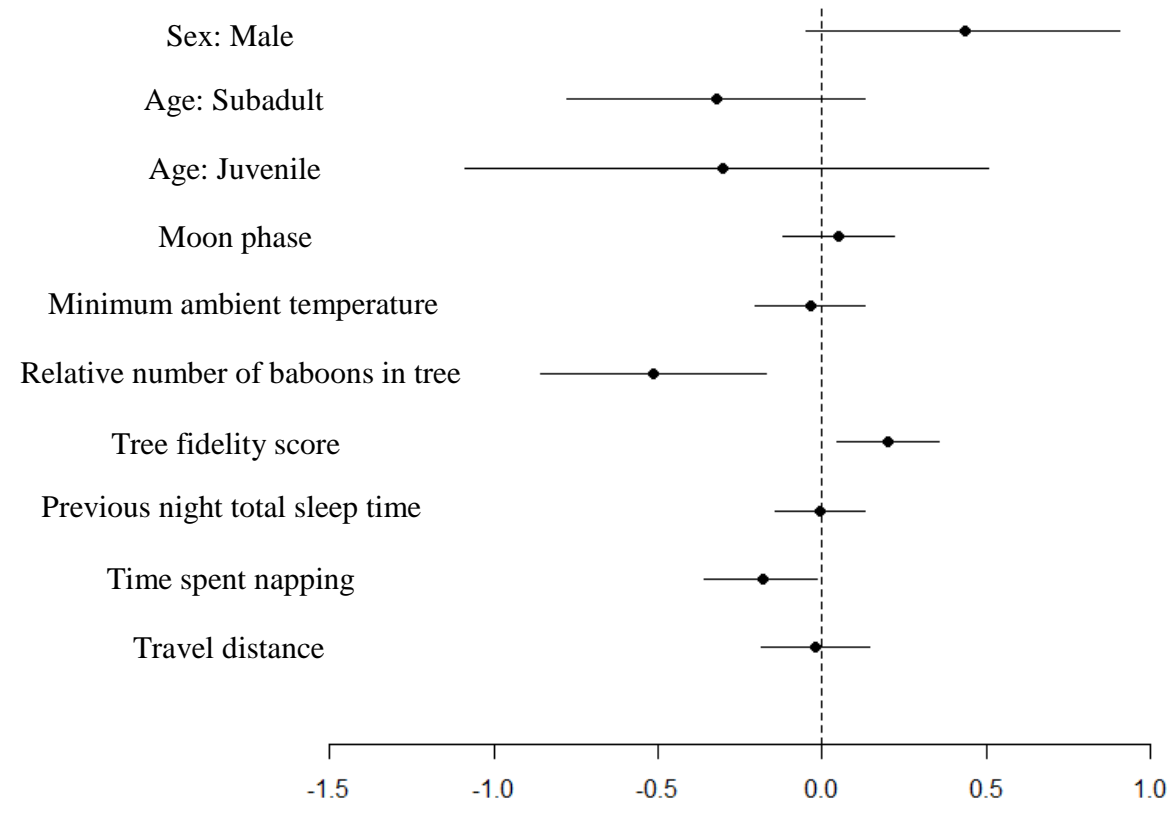

Figure S2. Model output plot of model of total sleep time (for the first 20 days) with all numerical variables standardized. The categorical variable tree is not plotted

\begin{tabular}{|l|c|c|}
\hline & \multicolumn{2}{c|}{ Total sleep time (hours) } \\
\hline Predictors & Estimates & CI (95\%) \\
\hline Intercept & 18.65 & $-24.20-63.97$ \\
\hline Travel distance $(\mathbf{k m})$ & -0.01 & $-0.12-0.09$ \\
\hline Time spent napping (mins) & -0.01 & $-0.01--0.00$ \\
\hline Previous night relative total sleep time (mins) & -0.00 & $-0.00-0.00$ \\
\hline Tree fidelity score & 0.60 & $0.15-1.03$ \\
\hline Relative number of baboons in tree & -1.55 & $-2.58--0.48$ \\
\hline Minimum ambient temperature (degree Celsius) & -0.03 & $-0.19-0.12$ \\
\hline Moon phase & 0.11 & $-0.25-0.48$ \\
\hline age: Juvenile & -0.26 & $-0.85-0.38$ \\
\hline age: Subadult & -0.25 & $-0.60-0.10$ \\
\hline sex: Male & 0.34 & $-0.03-0.72$ \\
\hline tree: tree2 & -0.20 & $-0.84-0.44$ \\
\hline tree: tree3 & -0.12 & $-0.79-0.55$ \\
\hline tree: tree4 & -0.64 & $-1.03--0.25$ \\
\hline tree: tree5 & -0.20 & $-0.59-0.19$ \\
\hline tree: tree6 & 0.02 & $-0.48-0.50$ \\
\hline tree: tree7 & 0.09 & $-0.46-0.64$ \\
\hline tree: tree8 & -0.22 & $-0.59-0.16$ \\
\hline tree: tree10 & -0.17 & $-0.74-0.38$ \\
\hline tree: tree11 & -0.18 & $-0.60-0.24$ \\
\hline Random Effects & & \\
\hline
\end{tabular}




\begin{tabular}{|l|l|}
\hline $\boldsymbol{\sigma}^{\mathbf{2}}$ & 0.31 \\
\hline $\boldsymbol{\tau}_{\mathbf{0 0}}$ night & 0.01 \\
\hline $\boldsymbol{\tau}_{\mathbf{0 0} \text { tag }}$ & 0.05 \\
\hline $\mathbf{I C C}$ & 0.17 \\
\hline $\mathbf{N}_{\text {tag }}$ & 18 \\
\hline $\mathbf{N}_{\text {night }}$ & 18 \\
\hline Observations & 170 \\
\hline Marginal R $\mathbf{R}^{\mathbf{2}}$ Conditional $\mathbf{R}^{\mathbf{2}}$ & $0.337 / 0.412$ \\
\hline
\end{tabular}

Table S4. Model output table of model total sleep time (for the first 20 days) with no standardization of variables

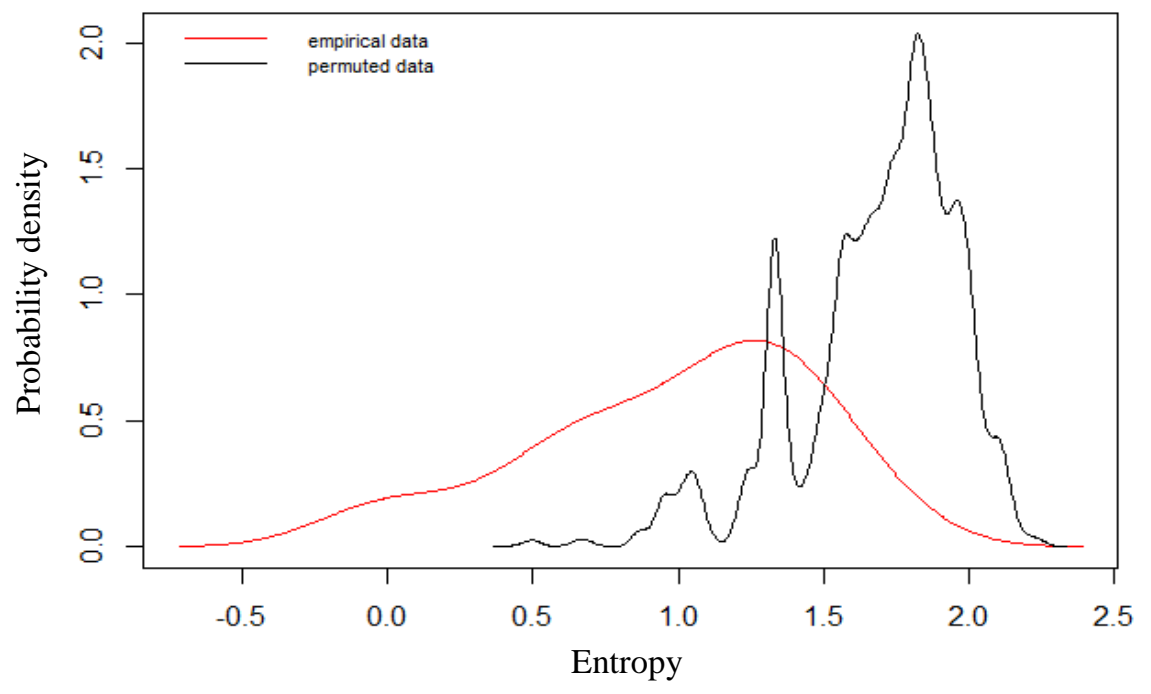

Figure S3. Comparison of the Shannon entropies of individuals' sleep tree occupancy within this sleep site to a null distribution produced by 1000 identity permutations. The analysis revealed lower entropy in tree occupancy than expected by random chance (one-tailed two-sample KolmogorovSmirnov test: $\mathrm{p}<1.0 \times 10^{-9}$ ), indicating that individuals exhibited high fidelity to particular trees. The red line represents the distribution of Shannon entropies of individuals' sleep tree occupancy calculated from the empirical data, and the black line represents the distribution of entropy of sleep tree occupancy derived from the permuted data set. 


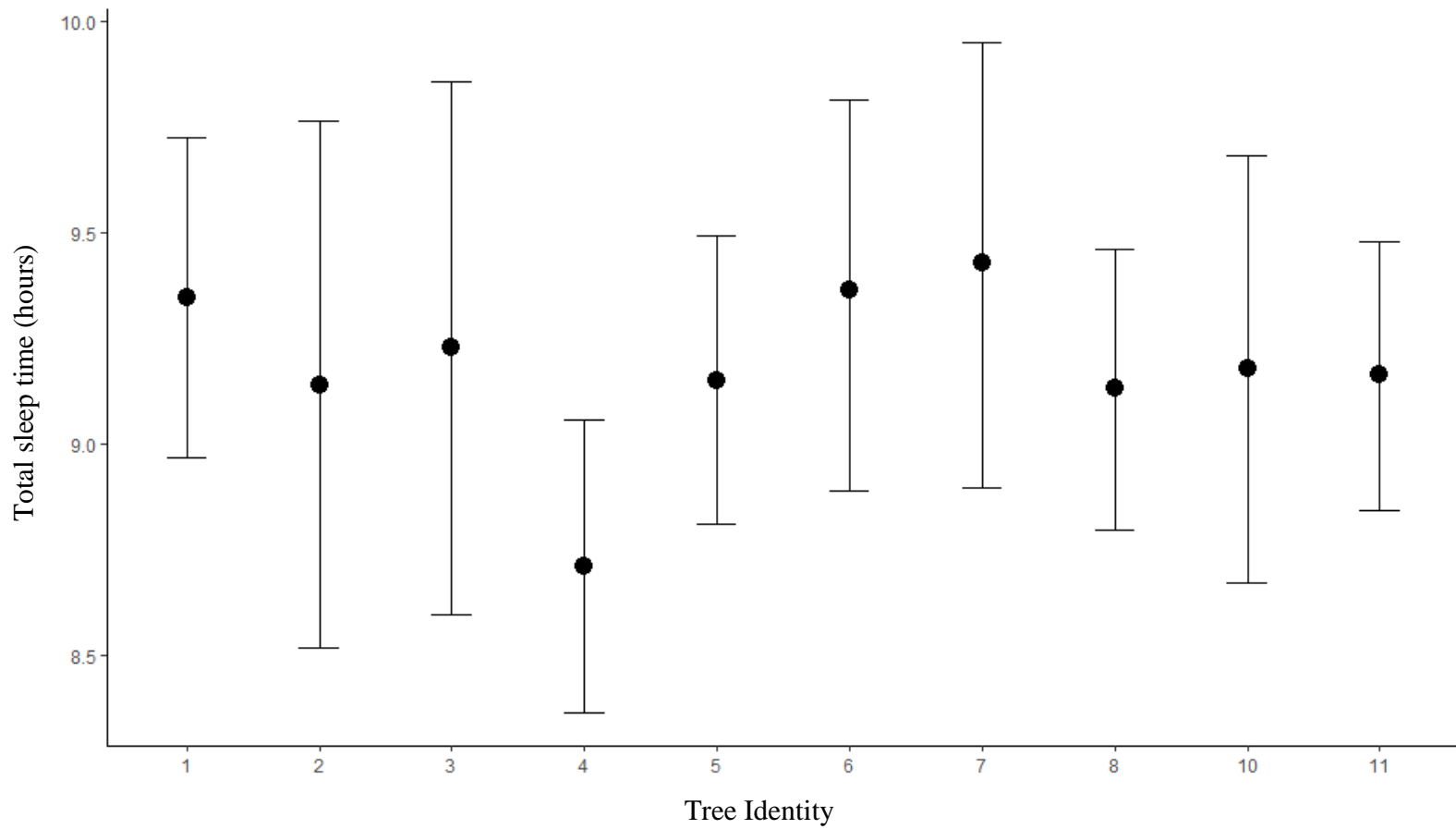

Figure S4. The conditional effect of tree identity on total sleep time. The conditional effects plotted here are from the unstandardized Bayesian linear mixed model (LMM) of total sleep time (hours).

\begin{tabular}{|c|c|c|}
\hline & \multicolumn{2}{|c|}{ Total sleep time (Standarized) } \\
\hline Predictors & Estimates & $C I(95 \%)$ \\
\hline Intercept & -0.04 & $-0.55-0.47$ \\
\hline Average VeDBA during day & -0.12 & $-0.33-0.12$ \\
\hline Time spent napping & -0.13 & $-0.30-0.05$ \\
\hline Previous night total sleep time & -0.00 & $-0.14-0.13$ \\
\hline Tree fidelity score & 0.20 & $0.04-0.36$ \\
\hline Relative number of baboons in tree & -0.55 & $-0.89--0.20$ \\
\hline Minimum ambient temperature & -0.04 & $-0.21-0.12$ \\
\hline Moon phase & 0.07 & $-0.09-0.23$ \\
\hline age: Juvenile & -0.21 & $-1.01-0.59$ \\
\hline age: Subadult & -0.26 & $-0.77-0.22$ \\
\hline sex: Male & 0.53 & $0.09-0.97$ \\
\hline tree: tree 2 & -0.34 & $-1.18-0.49$ \\
\hline tree: tree 3 & -0.47 & $-1.26-0.33$ \\
\hline tree: tree 4 & -0.86 & $-1.37--0.35$ \\
\hline tree: tree 5 & -0.28 & $-0.79-0.22$ \\
\hline tree: tree6 & 0.04 & $-0.60-0.68$ \\
\hline tree: tree 7 & 0.08 & $-0.66-0.83$ \\
\hline tree: tree8 & -0.28 & $-0.77-0.23$ \\
\hline tree: tree10 & -0.13 & $-0.84-0.58$ \\
\hline tree: tree11 & -0.24 & $-0.79-0.31$ \\
\hline \multicolumn{3}{|l|}{ Random Effects } \\
\hline$\sigma^{2}$ & \multicolumn{2}{|l|}{0.55} \\
\hline$\tau_{00 \text { night }}$ & \multicolumn{2}{|l|}{0.02} \\
\hline$\tau_{00 \text { tag }}$ & \multicolumn{2}{|l|}{0.07} \\
\hline ICC & \multicolumn{2}{|l|}{0.13} \\
\hline
\end{tabular}




\begin{tabular}{|l|l|}
\hline $\mathbf{N}_{\text {tag }}$ & 18 \\
\hline $\mathbf{N}_{\text {night }}$ & 18 \\
\hline Observations & 178 \\
\hline Marginal $\mathbf{R}^{2}$ / Conditional $\mathbf{R}^{\mathbf{2}}$ & $0.352 / 0.416$ \\
\hline
\end{tabular}

Table S5. Model output table of model total sleep time (for the first 20 days) with all numerical variables standardized

40

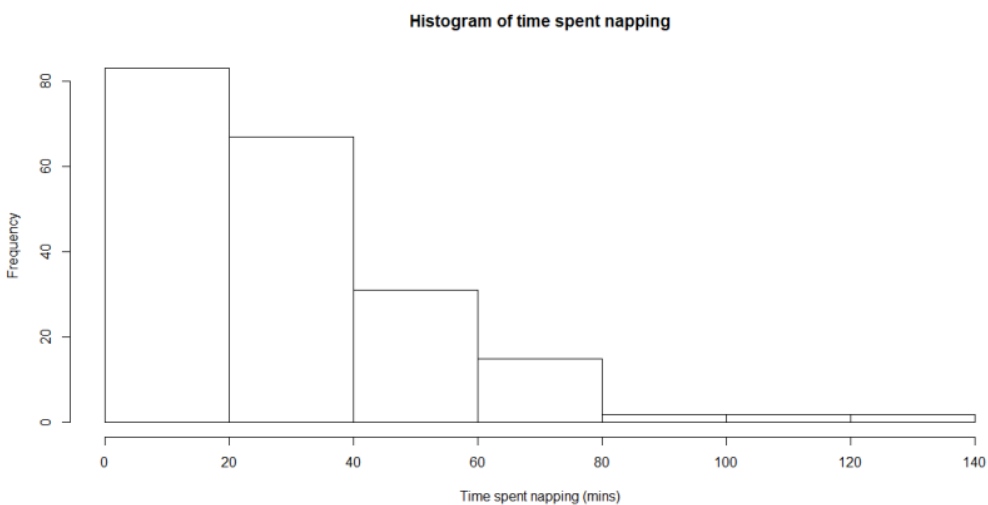

Figure S5. Histogram of time spent napping for the first 20 days of the study (data prior to leopard attack)

\begin{tabular}{|c|c|c|}
\hline & \multicolumn{2}{|c|}{ Time spent napping (standardized) } \\
\hline Predictors & Estimates & $C I(95 \%)$ \\
\hline Intercept & -0.10 & $-0.49-0.28$ \\
\hline Prior night total sleep time & 0.05 & $-0.08-0.18$ \\
\hline \multicolumn{3}{|l|}{ Random Effects } \\
\hline$\sigma^{2}$ & \multicolumn{2}{|l|}{0.41} \\
\hline$\tau_{00}$ night & \multicolumn{2}{|l|}{0.12} \\
\hline$\tau_{00 \text { tag }}$ & \multicolumn{2}{|l|}{0.51} \\
\hline ICC & \multicolumn{2}{|l|}{0.60} \\
\hline $\mathbf{N}_{\text {tag }}$ & \multicolumn{2}{|l|}{20} \\
\hline $\mathbf{N}_{\text {night }}$ & \multicolumn{2}{|l|}{19} \\
\hline Observations & \multicolumn{2}{|l|}{199} \\
\hline Marginal $\mathbf{R}^{2}$ / Conditional $\mathbf{R}^{2}$ & \multicolumn{2}{|l|}{$0.003 / 0.617$} \\
\hline
\end{tabular}

Table S6. Model output table of model of time spent napping during the day (for the first 20 days) with all numerical variables standardized

\begin{tabular}{|l|c|c|}
\hline & \multicolumn{2}{|c|}{ Time spent napping (minutes) } \\
\hline Predictors & Estimates & CI (95\%) \\
\hline Intercept & 21.22 & $-5.83-47.59$ \\
\hline Prior night total sleep time (hours) & 1.53 & $-1.23-4.42$ \\
\hline Random Effects & \multicolumn{2}{|l|}{} \\
\hline $\boldsymbol{\sigma}^{\mathbf{2}}$ & 295.93 \\
\hline $\boldsymbol{\tau}_{\mathbf{0 0} \text { night }}$ & 25.86 \\
\hline $\boldsymbol{\tau}_{\mathbf{0 0} \text { tag }}$ & 99.07 \\
\hline ICC & 0.30 \\
\hline $\mathbf{N}_{\text {tag }}$ & 20 \\
\hline $\mathbf{N}_{\text {night }}$ & 19 \\
\hline Observations & 199 \\
\hline
\end{tabular}




\section{Marginal $\mathbf{R}^{2}$ / Conditional $\mathbf{R}^{2}$}

$0.002 / 0.521$

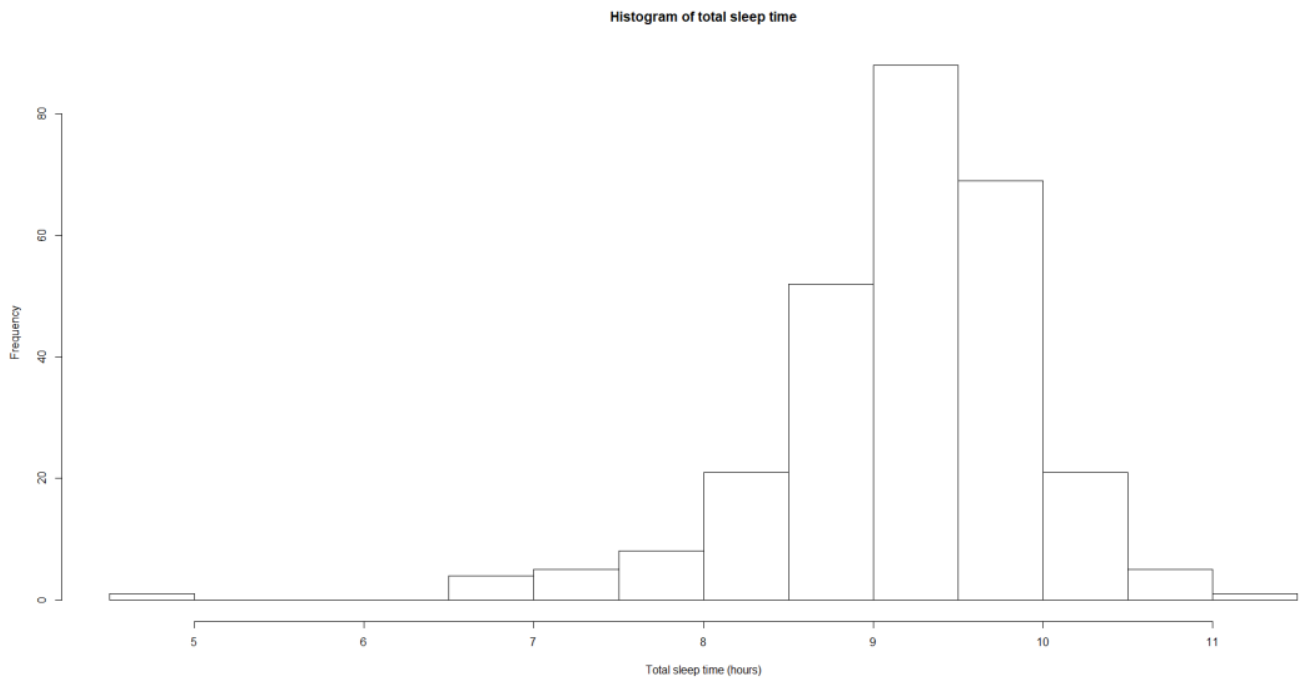

Figure S6. Histogram of total sleep time for the entire study duration

\begin{tabular}{|c|c|c|}
\hline & \multicolumn{2}{|c|}{ Total sleep time (standardized } \\
\hline Predictors & Estimates & $C I(95 \%)$ \\
\hline Intercept & -0.24 & $-0.57-0.11$ \\
\hline cond_night: night of leopard attack & -0.25 & $-0.87-0.39$ \\
\hline cond_night: first night in new sleep site & -1.55 & $-2.18--0.91$ \\
\hline cond_night: second night in new sleep site & -0.26 & $-0.98-0.44$ \\
\hline cond_night: third night in new sleep site & 0.21 & $-0.47-0.88$ \\
\hline cond_night: remainder of nights (in original sleep site) & 0.25 & $-0.07-0.56$ \\
\hline age: Juvenile & -0.27 & $-1.14-0.59$ \\
\hline age: Subadult & -0.36 & $-0.87-0.13$ \\
\hline sex: Male & 0.74 & $0.25-1.26$ \\
\hline Travel distance & -0.06 & $-0.18-0.07$ \\
\hline Time spent napping & -0.11 & $-0.28-0.05$ \\
\hline Previous night relative total sleep time & 0.15 & $0.04-0.27$ \\
\hline Minimum ambient temperature & -0.00 & $-0.14-0.14$ \\
\hline Moon phase & 0.02 & $-0.12-0.15$ \\
\hline \multicolumn{3}{|l|}{ Random Effects } \\
\hline$\sigma^{2}$ & \multicolumn{2}{|l|}{0.78} \\
\hline$\tau_{00 \text { tag }}$ & \multicolumn{2}{|l|}{0.14} \\
\hline ICC & \multicolumn{2}{|l|}{0.16} \\
\hline $\mathbf{N}_{\text {tag }}$ & \multicolumn{2}{|l|}{20} \\
\hline Observations & \multicolumn{2}{|l|}{275} \\
\hline Marginal $\mathbf{R}^{2}$ / Conditional $\mathbf{R}^{2}$ & \multicolumn{2}{|l|}{$0.251 / 0.314$} \\
\hline
\end{tabular}

Table S8. Model output table of model of total sleep time using data from entire study duration (including after the leopard attack) with all variables standardized

\section{Total sleep time (hours)}




\begin{tabular}{|c|c|c|}
\hline Intercept & 8.43 & $-43.81-59.58$ \\
\hline cond_night: night of leopard attack & -0.21 & $-0.90-0.48$ \\
\hline cond_night: first night in new sleep site & -1.19 & $-1.88--0.49$ \\
\hline cond_night: second night in new sleep site & -0.23 & $-0.98-0.48$ \\
\hline cond_night: third night in new sleep site & 0.15 & $-0.58-0.86$ \\
\hline cond_night: remainder of nights (in original sleep site) & 0.15 & $-0.19-0.48$ \\
\hline age: Juvenile & -0.28 & $-0.96-0.39$ \\
\hline age: Subadult & -0.31 & $-0.71-0.07$ \\
\hline sex: Male & 0.58 & $0.19-0.99$ \\
\hline Travel distance & -0.04 & $-0.13-0.05$ \\
\hline Time spent napping & -0.00 & $-0.01-0.00$ \\
\hline Previous night relative total sleep time & 0.00 & $0.00-0.00$ \\
\hline Minimum ambient temperature & 0.00 & $-0.17-0.19$ \\
\hline Moon phase & 0.07 & $-0.33-0.47$ \\
\hline \multicolumn{3}{|l|}{ Random Effects } \\
\hline$\sigma^{2}$ & \multicolumn{2}{|l|}{0.43} \\
\hline$\tau_{00}$ night & \multicolumn{2}{|l|}{0.05} \\
\hline$\tau_{00} \operatorname{tag}$ & \multicolumn{2}{|l|}{0.09} \\
\hline ICC & \multicolumn{2}{|l|}{0.25} \\
\hline $\mathbf{N}_{\text {tag }}$ & \multicolumn{2}{|l|}{20} \\
\hline $\mathbf{N}_{\text {night }}$ & \multicolumn{2}{|l|}{32} \\
\hline Observations & \multicolumn{2}{|l|}{275} \\
\hline Marginal $\mathbf{R}^{2}$ / Conditional $\mathbf{R}^{2}$ & \multicolumn{2}{|c|}{$0.257 / 0.365$} \\
\hline
\end{tabular}

Table S9. Model output table of model of total sleep time using data from entire study duration (including after the leopard attack) without standardization of variables

61

62
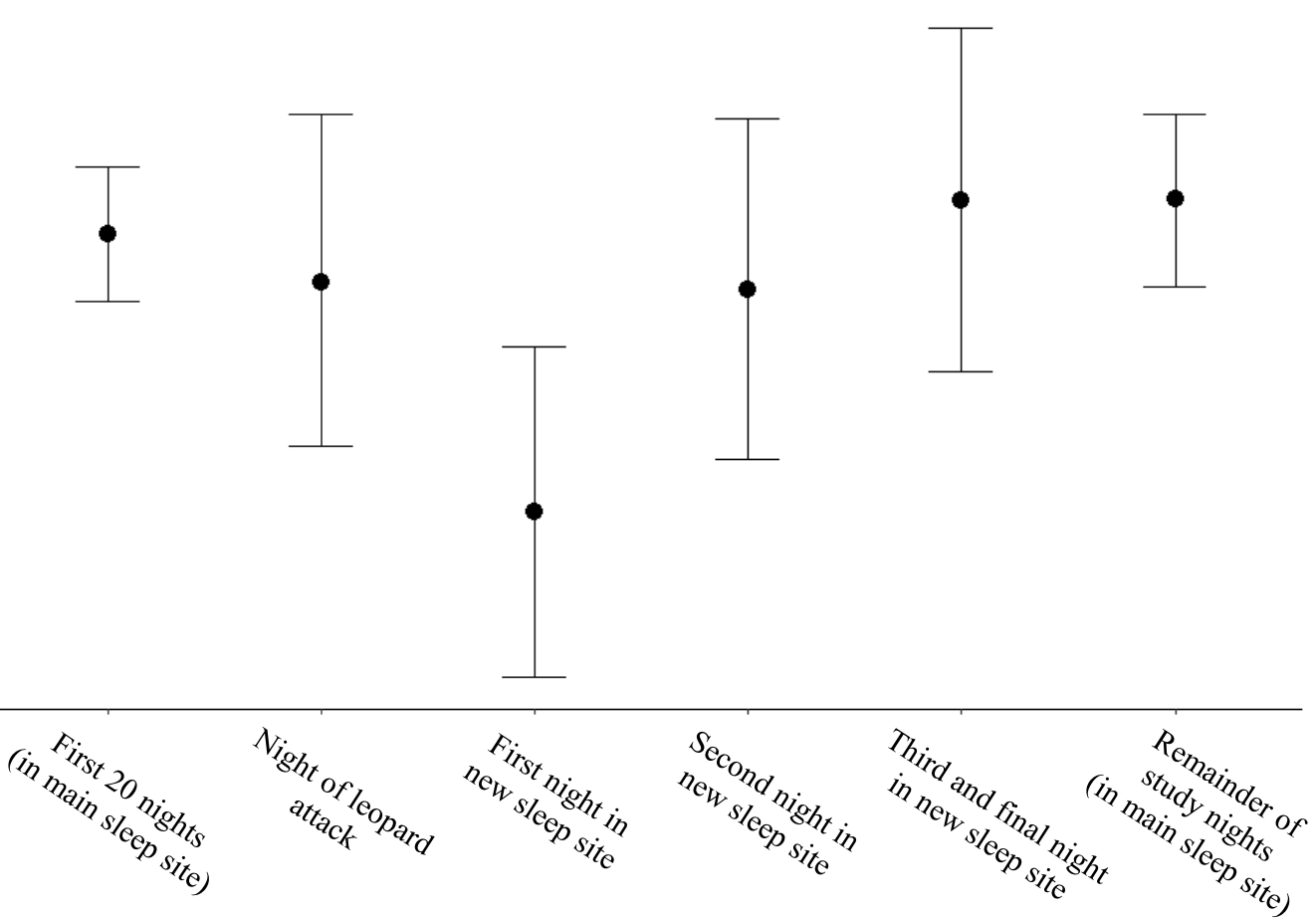

Figure S7. The conditional effect of night condition on total sleep time. The conditional effects presented here are from the unstandardized model of total sleep time. 


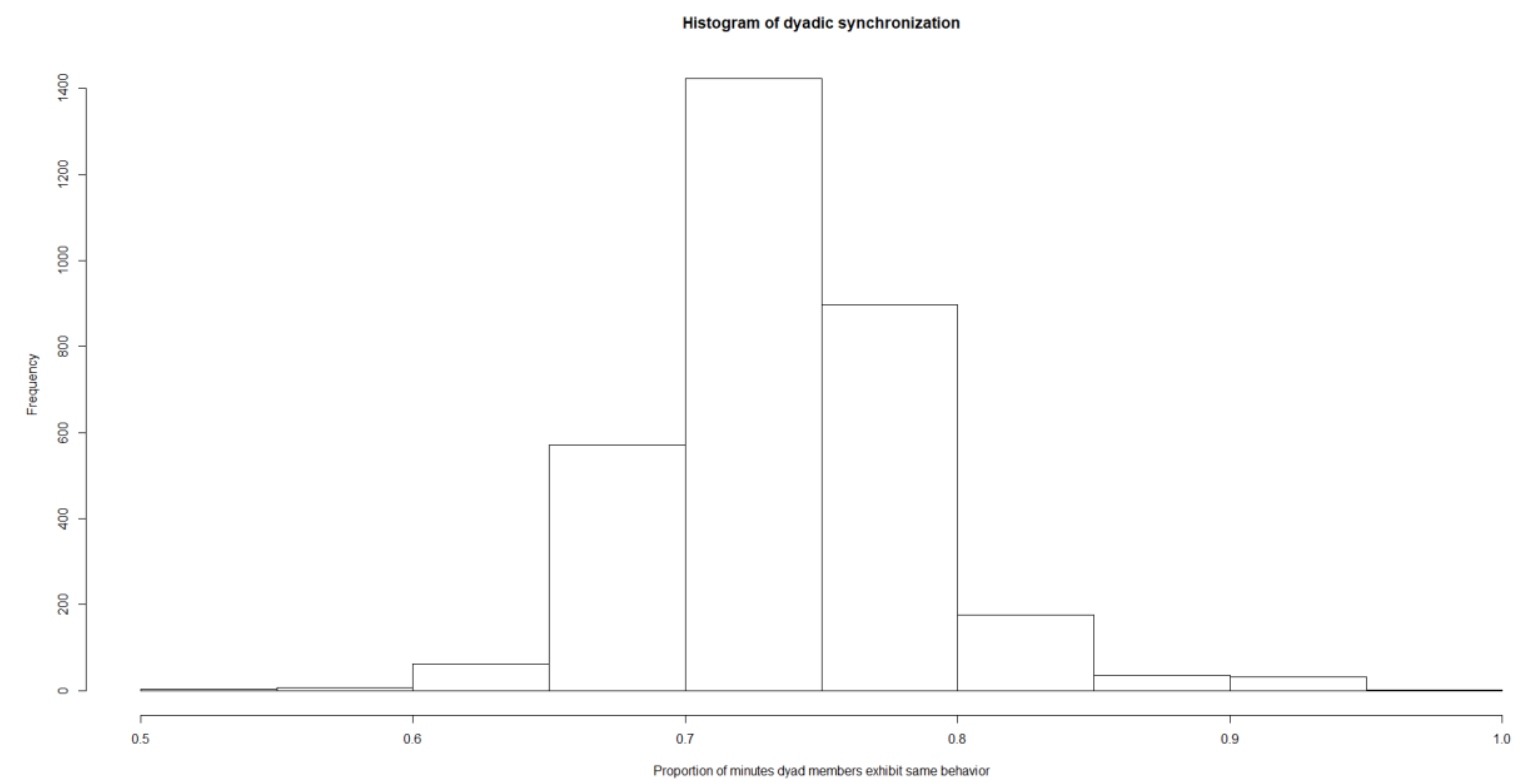

70 Figure S8. Histogram of the dyadic synchronization scores, which indicates the proportions of minutes between 21:00 and 05:00 during which both members of a dyad exhibited the same behavior (either sleep or wakefulness) on a given night.

\begin{tabular}{|c|c|c|}
\hline & \multicolumn{2}{|c|}{ Proportion of minutes synchronized (Standardized) } \\
\hline Predictors & Estimates & $C I(95 \%)$ \\
\hline Intercept & -0.21 & $-0.45-0.03$ \\
\hline Occupying same tree & 0.56 & $0.47-0.64$ \\
\hline \multicolumn{3}{|l|}{ Random Effects } \\
\hline$\sigma^{2}$ & 0.60 & \\
\hline$\tau_{00}$ dy_name & 0.14 & \\
\hline$\tau_{00 \text { night }}$ & 0.19 & \\
\hline$\tau_{00 \text { tag1 }}$ & 0.08 & \\
\hline$\tau_{00 \text { tag2 }}$ & 0.09 & \\
\hline $\mathrm{ICC}$ & 0.45 & \\
\hline $\mathbf{N}_{\text {night }}$ & 34 & \\
\hline $\mathbf{N}_{\text {tag1 }}$ & 22 & \\
\hline $\mathbf{N}_{\text {tag2 }}$ & 22 & \\
\hline $\mathbf{N}_{\text {dy_name }}$ & 250 & \\
\hline Observations & 2997 & \\
\hline Marginal $\mathbf{R}^{2}$ / Conditional $\mathbf{R}^{2}$ & $0.050 / 0.404$ & \\
\hline
\end{tabular}

Table S10. Model output table of model of synchronization (i.e. the proportion of minutes during a night that both dyad members exhibit the same behavior, either sleep or wakefulness) with response variable standardized of the response variable

\begin{tabular}{|l|c|c|}
\hline \multicolumn{2}{|c|}{ Proportion of minutes synchronized } \\
\hline Predictors & Estimates & CI (95\%) \\
\hline Intercept & 0.73 & $0.71-0.74$ \\
\hline Occupying same tree & 0.03 & $0.02-0.03$ \\
\hline Random Effects & \multicolumn{2}{|}{} \\
\hline $\boldsymbol{\sigma}^{\mathbf{2}}$ & 0.00 \\
\hline $\boldsymbol{\tau}_{\mathbf{0 0} \text { dy_name }}$ & 0.00 & \\
\hline $\boldsymbol{\tau}_{\mathbf{0 0} \text { night }}$ & 0.00 & \\
\hline
\end{tabular}


bioRxiv preprint doi: https://doi.org/10.1101/2021.09.26.461870; this version posted September 27, 2021. The copyright holder for this preprint (which was not certified by peer review) is the author/funder, who has granted bioRxiv a license to display the preprint in perpetuity. It is made available under aCC-BY 4.0 International license.

\begin{tabular}{|l|l|}
\hline $\boldsymbol{\tau}_{\mathbf{0 0} \text { tag1 }}$ & 0.00 \\
\hline $\boldsymbol{\tau}_{\mathbf{0 0} \text { tag2 }}$ & 0.00 \\
\hline $\mathbf{I C C}$ & 0.45 \\
\hline $\mathbf{N}_{\text {night }}$ & 34 \\
\hline $\mathbf{N}_{\text {tag1 }}$ & 22 \\
\hline $\mathbf{N}_{\text {tag2 }}$ & 22 \\
\hline $\mathbf{N}_{\text {dy_name }}$ & 250 \\
\hline Observations & 2997 \\
\hline Marginal $\mathbf{R}^{2} /$ Conditional $\mathbf{R}^{2}$ & $0.050 / 0.404$ \\
\hline
\end{tabular}

Table S11. Model output table of model of synchronization (i.e. the proportion of minutes during a night that both dyad members exhibit the same behavior, either sleep or wakefulness) without standardization of the response variable

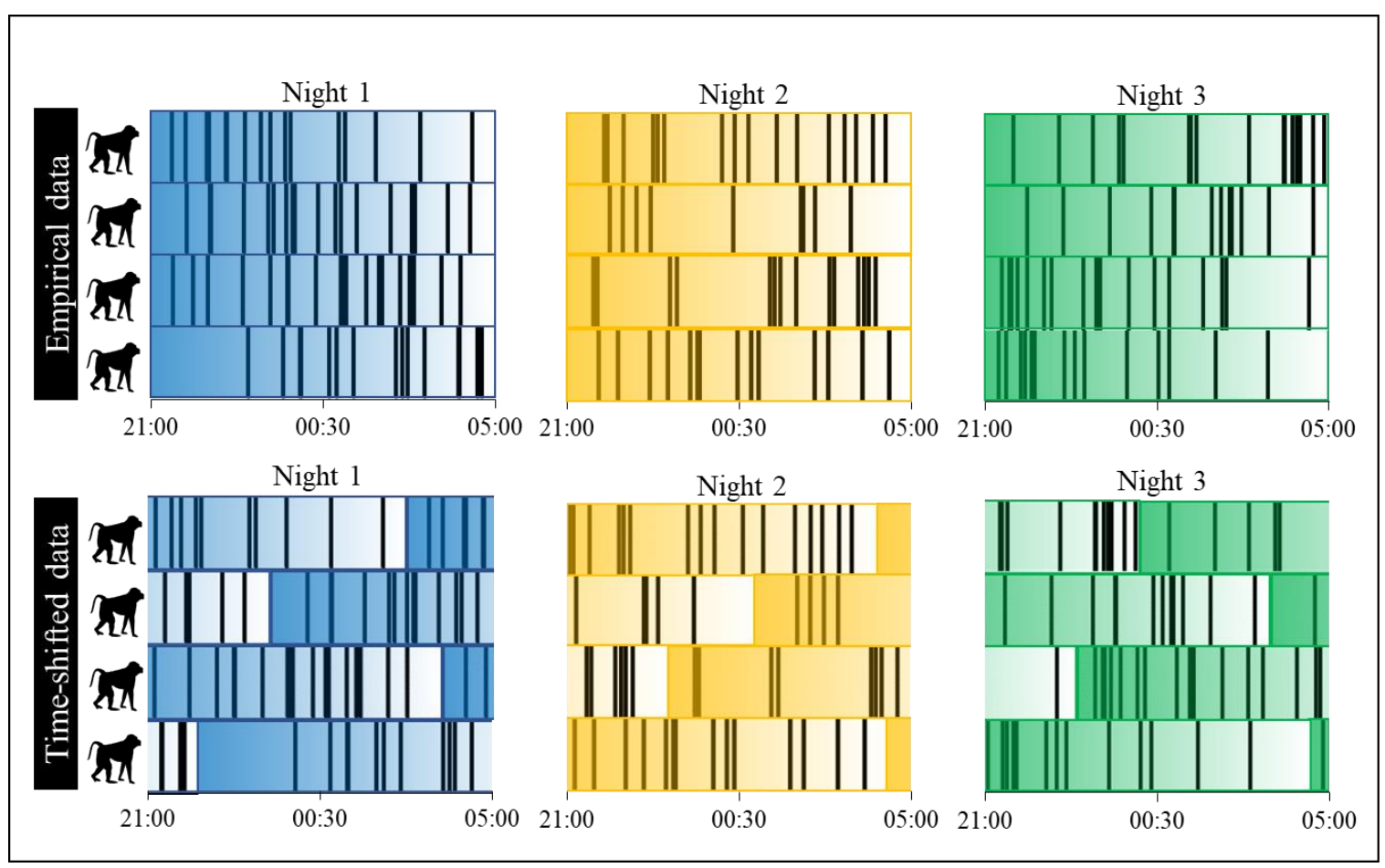

Figure S9. A toy example of the procedure we used to test for sentinel behavior and synchronization of nighttime behavior. Each row represents a baboon's time-series of sleep and wake activity during the night, with black vertical lines indicating periods of nocturnal waking behavior. Colors correspond to different nights, and the transparency of the color indicates the timing of night, with reference to the empirical, unshifted data. The time shifting procedure was repeated 1000 times to generate a null distribution for the proportion of minutes in which at least one individual is awake during the night and the mean proportion of the group exhibiting synchronized behavior. 


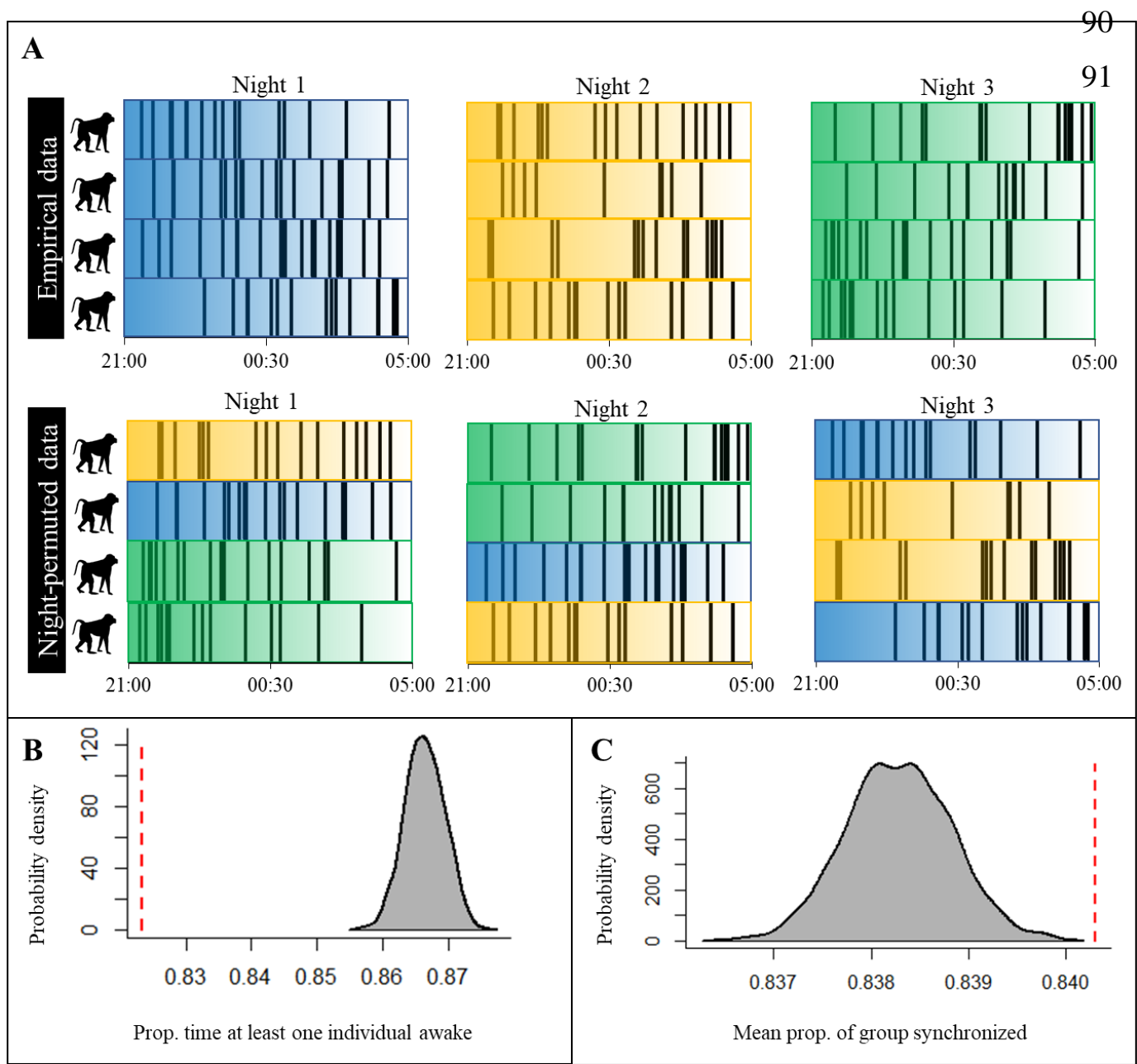

Figure S10. A) A toy example of the procedure alternative to the one presented in the main text (and represented in Figure S9) that we used to confirm findings concerning sentinel behavior and synchronization of nighttime behavior that we derived from the procedure presented in the main text. Each row represents a baboon's time-series of sleep and wake activity during the night, with black vertical lines indicating periods of nocturnal waking behavior. Colors correspond to different nights, with reference to the empirical, unpermuted data, and the transparency of the color indicates the timing of night. The night permutation procedure was repeated 1000 times to generate a null distribution for the proportion of minutes in which at least one individual is awake during the night and the mean proportion of the group exhibiting synchronized behavior. B) Comparison of the empirical proportion of minutes in which at least one individual is awake (red dotted line) to its null distribution (grey density plot; $p<0.0001$ ). C) Comparison of the empirical mean of the proportion of the group exhibiting synchronized behavior (red dotted line) to its null distribution (grey density plot; $\mathrm{p}<0.0001$ ). This method of permutation controls for the possibility that baboons are synchronized in their behavior simply as a result of species-typical nocturnal waking patterns that are consistent across baboons and across nights.

\section{Sleep validation study}

To evaluate whether the accelerometer-based sleep classification technique was accurately monitoring sleep in baboons, we returned to Mpala Research Centre in July 2019 to perform a validation study in which we compared the results of the accelerometer-based sleep classification to direct observations of awake and sleeping baboons. Using the procedures described in StrandburgPeshkin et al., 2015, we trapped and anesthetized 27 members of a group of habituated olive baboons, fitting each with a GPS and accelerometry collar. Eleven of the 27 collars deployed recorded continuous tri-axial accelerations at $12 \mathrm{~Hz} /$ axis from 06:30 to 18:00 and 0.71-second bursts of 
accelerations at $56.2 \mathrm{~Hz} / \mathrm{axis}$ at the beginning of every minute from 18:00 to 06:30. Accelerometry data was collected by each of these 11 collars for up to 31 days. The remaining 16 collars did not collect accelerometry data from 06:30 to 18:00, and thus we excluded data from these collars from the validation study.

We down-sampled and interpolated the accelerometry data such that it matched the sampling frequency and schedule of the data collected in 2012 (i.e. the data analyzed for this manuscript). We then applied the sleep classification algorithm described in the Materials and Methods to this validation dataset.

To validate the sleep classification algorithm, we performed direct behavioral observations of the baboons at their primary sleep site. We recorded the behavior of the study baboons starting when they approached their sleep site using a FLIR T1020 high-resolution infrared camera (FLIR Systems Inc., Wilsonville, OR, USA). Recordings continued into the night for as long as the camera battery allowed (average recording duration (range of recording durations): 7.4 hours (1.7 - 14.9 hours)), and we collected thermal imaging data on 21 nights. We identified individuals in the thermal imagery both in real-time, via observer narration of the recorded imagery, and post-recording, by matching movements of individuals in the thermal imagery to the GPS tracks of collared individuals.

Following initial data collection, we used the commercial software Loopy (Loopbio GmbH, Austria) to score the behavior of identified individuals in the thermal imagery. Individuals' behavior was scored as "wakefulness", "resting wakefulness", or "sleep". Wakefulness refers to any behavior involving active movement (i.e. walking, running) or engaged activity (i.e. allogrooming), whereas resting wakefulness refers to behaviors that are dormant (i.e. sitting), but not in the typical sleeping posture of a baboon (sitting or lying with neck relaxed and head hung). Sustained dormant behavior in the typical sleep posture was considered sleep. Video scoring resulted in a total of 8.0 hours of behavioral observation across a total of 16 individual baboons.

Synchronizing the thermal imagery data with the accelerometry data produced a validation dataset of 294 minute-epochs across six baboons that were both classified as either sleep or wakeful behavior from accelerometry, and scored as wakefulness, resting wakefulness, or sleep from direct observation. With both wakefulness and resting wakefulness representing wakeful behavior, the accelerometer-based sleep classification exhibited an accuracy of 79.9\% (Table S12). Consistent with previous validation studies of the use of accelerometry in measuring sleep (Ancoli-Israel et al., 2003; de Souza et al., 2003), we found that accelerometer-based sleep classification has difficulty distinguishing resting wakefulness from sleep, and we consider this limitation in our interpretation of the results.

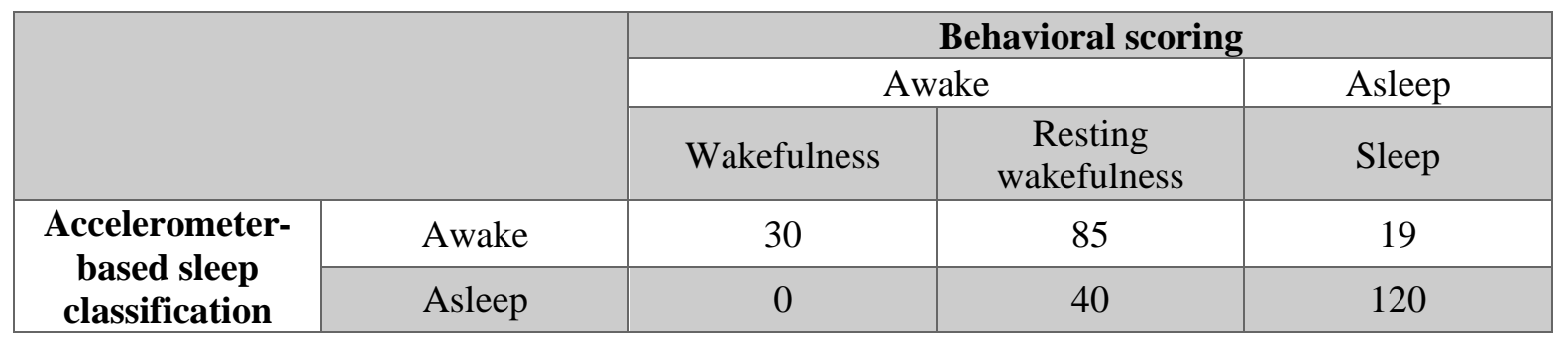

Table S12. Confusion matrix reporting the results of the validation study. Table entries represent the number of minute-epochs classified according to the accelerometer-based technique and direct behavioral observation. 